\title{
M-Current Inhibition in Hippocampal Excitatory Neurons Triggers Intrinsic and Synaptic Homeostatic Responses at Different Temporal Scales
}

\author{
Jonathan Lezmy, ${ }^{\star}$ Hana Gelman, ${ }^{\star}$ Maxim Katsenelson, Boaz Styr, Eliav Tikochinsky, Maya Lipinsky, \\ Asher Peretz, Inna Slutsky, and Bernard Attali \\ Department of Physiology and Pharmacology, Sackler Faculty of Medicine and Sagol School of Neurosciences, Tel Aviv University, Tel Aviv 69978, \\ Israel
}

Persistent alterations in neuronal activity elicit homeostatic plastic changes in synaptic transmission and/or intrinsic excitability. However, it is unknown whether these homeostatic processes operate in concert or at different temporal scales to maintain network activity around a set-point value. Here we show that chronic neuronal hyperactivity, induced by M-channel inhibition, triggered intrinsic and synaptic homeostatic plasticity at different timescales in cultured hippocampal pyramidal neurons from mice of either sex. Homeostatic changes of intrinsic excitability occurred at a fast timescale (1-4 h) and depended on ongoing spiking activity. This fast intrinsic adaptation included plastic changes in the threshold current and a distal relocation of FGF14, a protein physically bridging $\mathrm{Na}_{\mathrm{v}} 1.6$ and $\mathrm{K}_{\mathrm{v}} 7.2$ channels along the axon initial segment. In contrast, synaptic adaptations occurred at a slower timescale $(\sim 2 \mathrm{~d})$ and involved decreases in miniature EPSC amplitude. To examine how these temporally distinct homeostatic responses influenced hippocampal network activity, we quantified the rate of spontaneous spiking measured by multielectrode arrays at extended timescales. M-Channel blockade triggered slow homeostatic renormalization of the mean firing rate (MFR), concomitantly accompanied by a slow synaptic adaptation. Thus, the fast intrinsic adaptation of excitatory neurons is not sufficient to account for the homeostatic normalization of the MFR. In striking contrast, homeostatic adaptations of intrinsic excitability and spontaneous MFR failed in hippocampal GABAergic inhibitory neurons, which remained hyperexcitable following chronic M-channel blockage. Our results indicate that a single perturbation such as M-channel inhibition triggers multiple homeostatic mechanisms that operate at different timescales to maintain network mean firing rate.

Key words: axon initial segment; homeostatic plasticity; M-channels; potassium channel; synaptic plasticity

Significance Statement

Persistent alterations in synaptic input elicit homeostatic plastic changes in neuronal activity. Here we show that chronic neuronal hyperexcitability, induced by M-type potassium channel inhibition, triggered intrinsic and synaptic homeostatic plasticity at different timescales in hippocampal excitatory neurons. The data indicate that the fast adaptation of intrinsic excitability depends on ongoing spiking activity but is not sufficient to provide homeostasis of the mean firing rate. Our results show that a single perturbation such as $\mathrm{M}$-channel inhibition can trigger multiple homeostatic processes that operate at different timescales to maintain network mean firing rate.

Received Aug. 7, 2019; revised Mar. 10, 2020; accepted Mar. 11, 2020.

Author contributions: J.L., I.S., and B.A. designed research; J.L., H.G., M.K., B.S., E.T., M.L., and A.P. performed research; J.L., H.G., M.K., B.S., E.T., M.L., A.P., I.S., and B.A. analyzed data; J.L., I.S., and B.A. wrote the paper.

*J.L. and H.G. contributed equally to this work.

The authors declare no competing financial interests.

This work was supported by a grant from the Israel Science Foundation (ISF 1365/17) to B.A. and by research grants from the European Research Council to I.S. (724866). B.A. holds the Andy Libach Professorial

Chair in clinical pharmacology and toxicology. We thank llana Shapira for excellent technical assistance.

Correspondence should be addressed to Bernard Attali at battali@tauex.tau.ac.il.

https://doi.org/10.1523/JNEUROSCI.1914-19.2020

Copyright $\odot 2020$ the authors

\section{Introduction}

Preserving the delicate balance between stability and adaptability, neurons use various powerful mechanisms to stabilize firing rates at defined set-point values in response to bidirectional perturbations of the network activity (Turrigiano et al., 1998; Abbott and Nelson, 2000; Burrone et al., 2002; Desai et al., 2002; Turrigiano and Nelson, 2004; Davis, 2006; Marder and Goaillard, 2006; Turrigiano, 2011; Slomowitz et al., 2015; Styr and Slutsky, 2018). These homeostatic changes include synaptic adaptations and/ or changes in intrinsic neuronal excitability. The homeostatic regulation of synaptic function includes modifications of 
synaptic strength, excitation-to-inhibition (E/I) balance, synapse number, and structure (Turrigiano, 2012; Yin and Yuan, 2014; Wefelmeyer et al., 2016). The homeostatic regulation of intrinsic excitability involves alterations in ion channels expression and in the structural organization of the axon initial segment (AIS; Turrigiano, 2011, 2012; Yoshimura and Rasband, 2014; Kole and Brette, 2018).

Little is known about the interaction between the synaptic and intrinsic homeostatic plasticity. The occurrence of intrinsic and synaptic adaptations was in some cases dependent on the developmental stage, brain region, and type of neurons. For example, in the primary sensory cortex, visual deprivation during the precritical period induced synaptic upscaling of excitatory synapses onto layer 4 pyramidal neurons and reduction of inhibition, but no changes in intrinsic excitability (Desai et al., 2002; Maffei et al., 2004). In contrast, lid suture during the classical critical period triggered intrinsic, but not synaptic homeostatic plasticity in the layer 2/3 pyramidal neurons (Desai et al., 2002; Goel and Lee, 2007; Maffei and Turrigiano, 2008). In the hippocampus, longterm whisker deprivation triggered synaptic and intrinsic adaptations during early development, while only intrinsic excitability was affected in adult mice (Milshtein-Parush et al., 2017). In primary neuronal cultures, chronic inactivity triggered both synaptic and intrinsic adaptive responses (Desai, 2003; Slomowitz et al., 2015; Joseph and Turrigiano, 2017). Interestingly, in hippocampal organotypic slices, changes in intrinsic excitability preceded those in synaptic strength (Karmarkar and Buonomano, 2006). More recently, it was shown in cultured cortical neurons that excitatory synaptic scaling and intrinsic excitability are tightly coordinated through bidirectional changes in the same signaling pathway involving calcium/calmodulin-dependent protein kinase IV (Joseph and Turrigiano, 2017).

Despite the compelling evidence for the existence of various synaptic and intrinsic homeostatic plasticity mechanisms, crucial questions remain unaddressed. When both intrinsic and synaptic adaptations occur, do they operate at similar timescales? Do intrinsic adaptations require ongoing spiking activity, or are they spike independent? Are inhibitory neurons undergoing similar homeostatic plasticity changes as excitatory neurons following a similar hyperexcitability trigger? To address these questions, we examined the intrinsic and synaptic homeostatic adaptations induced by chronic M-channel blockade in cultured mouse hippocampal neurons using whole-cell patch-clamp electrophysiology and microelectrode arrays (MEAs) for extracellular spike recordings, allowing the same neurons to be recorded over long timescales (Slomowitz et al., 2015). Here, we show that chronic M-channel inhibition by the specific blocker XE991 triggered a fast, activity-dependent homeostatic intrinsic adaptation and a slow synaptic homeostatic plasticity in pyramidal excitatory neurons. Using an adeno-associated virus (AAV) vector driving the expression of the fluorescent protein mCherry under the control of the specific GABAergic hDlx promoter (Dimidschstein et al., 2016), patch-clamp recording of inhibitory hippocampal neurons showed that following chronic M-channel block, no homeostatic plasticity of intrinsic excitability and spontaneous mean firing rate (MFR) was observed. In sharp contrast to excitatory neurons, hippocampal GABAergic neurons remained hyperexcitable. We conclude that the fast intrinsic adaptation of excitatory neurons is not sufficient to account for the homeostatic normalization of the MFR and that the same perturbation such as M-channel inhibition can trigger multiple homeostatic mechanisms that operate at different timescales to maintain network activity.

\section{Materials and Methods}

Animals. BALB/c mice of either sex were used for generating the primary cultures of hippocampal neurons. All experimental protocols conformed to the guidelines of the Institutional Animal Care and Use Committee of Tel Aviv University (Tel Aviv, Israel), and to the guidelines of the National Institutes of Health (animal welfare authorization no. 01-16-012).

Drugs. XE991 dihydrochloride (catalog \#2000/10, Tocris Bioscience), 1(S),9(R)-(-)-bicuculline methiodide (catalog \#14343, Sigma-Aldrich), picrotoxin (catalog \#P1675, Sigma-Aldrich), tetrodotoxin (TTX) citrate (catalog \#T-550, Alomone Labs), NBQX hydrate (catalog \#N171, SigmaAldrich), AP-5-DL-2-amino-5-phosphonopentanoic acid (catalog \#A5282, Sigma-Aldrich), and QX314Br (Alomone Labs).

Recombinant $A A V$-Dlx-mCherry plasmid and infection. Recombinant $\mathrm{AAV}$-virus-Dlx-mCherry plasmid was prepared by inserting the $\mathrm{hDlx}$ promoter sequence $(541 \mathrm{bp})$ upstream of the coding sequence in the backbone of the pAAV2-mCherry plasmid. Sequence identity was verified by routine DNA sequencing. The Dlx promoter was shown to restrict reporter expression in vivo to all GABAergic interneurons in the forebrain, including hippocampus, as well as in cultured neurons in vitro (Dimidschstein et al., 2016). The recombinant AAV2-virus-Dlx-mCherry was produced using standard production methods in HEK293 cells. All batches produced were in the range of 109-1010 viral particles $/ \mathrm{ml}$. Infections of hippocampal cultures were performed at $9 \mathrm{~d}$ in vitro (DIV), and recording was conducted at 14-16 DIV.

Primary cultures of hippocampal neurons. Hippocampi were dissected out from neonate BALB/c mice brains of either sex (0-1 d old). Hippocampi were washed three times in a HBSS-based solution containing the following: $4 \mathrm{~mm} \mathrm{NaHCO} 3,5 \mathrm{~mm}$ HEPES, and HBSS (SigmaAldrich), $\mathrm{pH}$ adjusted to $7.3-7.4$ at $4^{\circ} \mathrm{C}$. Tissues were digested in a solution including the following: $137 \mathrm{~mm} \mathrm{NaCl}, 5 \mathrm{~mm} \mathrm{KCl}, 7 \mathrm{mM} \mathrm{Na}_{2} \mathrm{HPO}_{4}$, $25 \mathrm{~mm}$ HEPES, $4.45 \mathrm{mg} / \mathrm{ml}$ trypsin type XI (Sigma-Aldrich), and 1614 $\mathrm{U} / \mathrm{ml}$ DNase type IV (Sigma-Aldrich), $\mathrm{pH}$ adjusted to 7.2 at $4^{\circ} \mathrm{C}$. Hippocampal tissues were incubated for $15 \mathrm{~min}$ in $37^{\circ} \mathrm{C}$ and washed again once with $5 \mathrm{ml} \mathrm{HBSS} / 20 \%$ FBS (fetal bovine serum) and once with HBSS. The cells were dissociated in an HBSS solution including 13.15 mM $\mathrm{MgSO}_{4}$ and $1772 \mathrm{U} / \mathrm{ml}$ DNase type IV (Sigma-Aldrich). Next, the cells were mechanically triturated with fire-polished Pasteur pipettes. $\mathrm{HBSS} / 20 \% \mathrm{FBS}$ was added to the dissociated cells, and the mixture was centrifuged at $1000 \times \mathrm{g}$, at $4^{\circ} \mathrm{C}$ for $10 \mathrm{~min}$. The supernatant was discarded and a plating medium including, MEM (Invitrogen), $24.7 \mathrm{~mm}$ glucose, $0.089 \mathrm{mg} / \mathrm{ml}$ transferrin (Calbiochem), glutamax (Sigma-Aldrich), $0.75 \mathrm{U} / \mathrm{ml}$ insulin (Sigma-Aldrich), 10\% FBS (Biological Industries), and B27 (Invitrogen), was added to the pellet. The cells were resuspended in the plating medium with fire-polished Pasteur pipette, and drops were added to glass coverslips coated with Matrigel in 24-well plates. After $1 \mathrm{~h}$ of incubation at $37^{\circ} \mathrm{C}$, the plating medium was added to the wells. The day after plating and twice a week, half of the medium was removed from the wells and replaced with the same volume of a feeding medium (MEM: $26.92 \mathrm{mM}$ glucose, $0.097 \mathrm{mg} / \mathrm{ml}$ transferrin, glutamax, B-27, and $3 \mu \mathrm{M}$ Ara-C; SigmaAldrich).

Acute hippocampal slices. Three-week-old male BALB/c mice were anesthetized and decapitated, and the brains were removed using procedures approved by the guidelines of the Institutional Animal Care and Use Committee of Tel Aviv University. Horizontal slices (400 $\mu \mathrm{M}$ ) were cut with a Vibratome (VT1200, Leica) in an oxygenated solution at $4^{\circ} \mathrm{C}$ containing the following: $1.25 \mathrm{~mm} \mathrm{NaH}_{2} \mathrm{PO}_{4}, 2.5 \mathrm{~mm} \mathrm{KCl}, 7 \mathrm{~mm} \mathrm{MgCl}$, $25 \mathrm{~mm}$ NaHCO3, $25 \mathrm{~mm}$ glucose, $87 \mathrm{~mm} \mathrm{NaCl}, 75 \mathrm{~mm}$ sucrose, and 0.5 $\mathrm{mm} \mathrm{CaCl} 2$. The slices were transferred to an oxygenated ACSF solution at $37^{\circ} \mathrm{C}$ for $45 \mathrm{~min}$ and then kept at room temperature.

Patch-clamp electrophysiology. Patch clamp was performed in the whole-cell configuration. Signals were filtered at $2 \mathrm{kHz}$ and digitized at $10 \mathrm{kHz}$ for miniature EPSCs (mEPSCs) or $5 \mathrm{kHz}$ for the other recordings. Signals were amplified using Axopatch 200B (Molecular Devices) for whole-cell patch recordings of cultured hippocampal neurons and Axopatch 700B for hippocampal slices recordings. In the experiments of chronic XE991 exposure involving recordings at different time points 
(1-48 h), untreated cells were also recorded to survey any possible variability, but no particular variance was observed in control untreated cells within the recording time window (1-48 h). For recordings in the current-clamp configuration, the extracellular solution contained $160 \mathrm{~mm}$ $\mathrm{NaCl}, 2.5 \mathrm{~mm} \mathrm{KCl}, 10 \mathrm{~mm}$ HEPES, $10 \mathrm{~mm}$ glucose, $1.2 \mathrm{~mm} \mathrm{Mg}^{2+}$, and 1.8 $\mathrm{mm} \mathrm{Ca}{ }^{2+}$, with $\mathrm{pH}$ adjusted to 7.3 with $\mathrm{NaOH}$. Microelectrodes with resistances of 5-8 M $\Omega$ were pulled from borosilicate glass capillaries (Harvard Apparatus) and filled with an intracellular solution. The intracellular solution contained the following: $135 \mathrm{~mm} \mathrm{KCl,} 1$ mм KATP, 1 mм MgATP, 2 mм EGTA, $1.1 \mathrm{~mm} \mathrm{CaCl} 2,10 \mathrm{~mm}$ HEPES, and $5 \mathrm{~mm}$ glucose, with $\mathrm{pH}$ adjusted to 7.25 with $\mathrm{KOH}$. For the experiments inspecting the threshold current and the resting membrane potential (RMP) of dissociated neurons, the following synaptic blockers were added to the extracellular solution to prevent spontaneous spikes: $30 \mu \mathrm{M}$ picrotoxin, $10 \mu \mathrm{M}$ bicuculline, $10 \mu \mathrm{M}$ NBQX, and $10 \mu \mathrm{M}$ AP-5. For the recording of spontaneous EPSCs (sEPSCs) and spontaneous IPSCs (sIPSCs), the same extracellular solution was used and contained, respectively, either $30 \mu \mathrm{M}$ picrotoxin $+10 \mu \mathrm{m}$ bicuculline or $10 \mu \mathrm{M}$ NBQX $+10 \mu \mathrm{M}$ AP-5. The intracellular solution contained the following: $0.4 \mathrm{~mm}$ GTP, $4 \mathrm{~mm}$ Na2ATP, $127 \mathrm{~mm} \mathrm{CsOH}, 127 \mathrm{~mm}$ D-gluconic acid, $4 \mathrm{~mm} \mathrm{CsCl}, 10 \mathrm{~mm}$ HEPES, $8 \mathrm{~mm} \mathrm{NaCl}$, and $0.4 \mathrm{~mm}$ EGTA, with $\mathrm{pH}$ adjusted to 7.25 with $\mathrm{CsOH}$. The $5 \mathrm{~mm} \mathrm{QX} 314 \mathrm{Br}$ was added to the intracellular solution before recordings. To isolate sEPSCs and sIPSCs on the same cell, the voltage was held on the reversal potential of $\mathrm{GABA}_{\mathrm{A}}(-45 \mathrm{mV})$ and AMPA $(+5 \mathrm{mV})$ receptors, respectively. The reversal potentials were calculated and experimentally verified with the recording solutions before the experiments with XE991. Liquid junction potential was calculated and subtracted from the recorded voltage $(+5$ and $+17 \mathrm{mV}$ were subtracted, respectively, for recordings of cultured hippocampal neurons and of CA1 pyramidal neurons from slices). The reversal potential of $\mathrm{GABA}_{\mathrm{A}}$ was notably depolarized because of the intracellular addition of bromide ions together with $\mathrm{QX} 314$ as $\mathrm{GABA}_{\mathrm{A}}$ was shown to be permeable to $\mathrm{Br}^{-}$. For mEPSCs recordings, the extracellular solution contained $160 \mathrm{~mm} \mathrm{NaCl}, 2.5 \mathrm{~mm} \mathrm{KCl}, 10 \mathrm{~mm}$ HEPES, $10 \mathrm{~mm}$ glucose, and $2 \mathrm{~mm}$ $\mathrm{CaCl} 2$, with $\mathrm{pH}$ adjusted to 7.3 with $\mathrm{NaOH}$. The $1 \mu \mathrm{M}$ tetrodotoxin, 30 $\mu \mathrm{M}$ picrotoxin, and $10 \mu \mathrm{m}$ bicuculline were added to the extracellular solution before the experiments. The intracellular solution contained 125 mм CsMeSO3, $15 \mathrm{~mm} \mathrm{CsCl,} 10 \mathrm{~mm}$ HEPES, $0.5 \mathrm{~mm} \mathrm{CaCl} 2,3 \mathrm{~mm}$ $\mathrm{MgCl} 2,3 \mathrm{~mm}$ Na4BAPTA, and $2 \mathrm{~mm}$ Na2ATP, with $\mathrm{pH}$ adjusted to 7.2 with $\mathrm{CsOH}$. mEPSCs recordings were performed while holding the voltage at $-70 \mathrm{mV}$, and the patch-pipettes were coated with Sylgard (Dow Corning). Recordings from hippocampal slices were performed in ACSF, as follows: $125 \mathrm{~mm} \mathrm{NaCl}, 1.25 \mathrm{~mm} \mathrm{NaH}_{2} \mathrm{PO}_{4}, 26 \mathrm{~mm} \mathrm{NaHCO} 3,3$ $\mathrm{mm} \mathrm{KCl}, 10 \mathrm{~mm}$ glucose, $2 \mathrm{~mm} \mathrm{MgCl}$, and $2 \mathrm{~mm} \mathrm{CaCl} 2$. The intracellular solution for slice recording contained the following: $145 \mathrm{~mm} \mathrm{~K}$-gluconate, $2 \mathrm{~mm} \mathrm{MgCl} 2$, $0.5 \mathrm{~mm}$ EGTA, 2 mм ATP-Tris, $0.2 \mathrm{~mm}$ Na2GTP, and $10 \mathrm{~mm}$ HEPES. CA1 pyramidal neurons from acute slices do not fire spontaneously in these conditions, therefore no synaptic blockers were added to the extracellular solution. For prolonged recordings, only patch-clamp cells with access resistance of $<30 \mathrm{M} \Omega$ that did not vary by $>10 \%$ throughout the experiment were recorded. All the electrophysiological experiments were performed at room temperature.

Immunostaining. XE991 was added to hippocampal neurons from primary culture for 4 or $48 \mathrm{~h}$ prior to staining, meaning that the cells (control and treated) were at the same age when fixed for immunostaining (16 DIV). For staining of hippocampal cultures, neurons were fixed in $4 \%$ paraformaldehyde for $10 \mathrm{~min}$ and washed three times in PBS. Permeabilization of the membrane was performed by adding $0.1 \%$ Triton X-100 (Sigma-Aldrich) in a blocking solution (PBS with $0.1 \%$ BSA and 5\% goat serum) for 4 min. After washing once with PBS, blocking solution was added to the coverslips for $10 \mathrm{~min}$. The primary antibodies were added to the neurons over $1 \mathrm{~h}$ at room temperature. The coverslips were washed twice with PBS for $10 \mathrm{~min}$ and incubated at room temperature over $1 \mathrm{~h}$ with the secondary antibodies. After washing twice with PBS, the coverslips were mounted in Fluoromount (SigmaAldrich). The primary antibodies used for immunostaining were as follows: mouse $\alpha$-FGF14 (1:500; clone N56/21, catalog \#75096, lot 4138RR-61, NeuroMab); rabbit $\alpha$-MAP2 (1:1000; catalog \#AB5622, lot 2795016, Millipore); and rabbit $\alpha$-VGAT (1:500; catalog \#131002,
Synaptic Systems). The secondary antibodies used against the primary antibodies were as follows: Invitrogen goat $\alpha$-mouse Alexa Fluor 488 (1:1000; Thermo Fisher Scientific; RRID:AB_2536161); donkey $\alpha$-rabbit Cy3 (1:500; catalog \#711-165-152, lot 143460, The Jackson Laboratory); and goat $\alpha$-mouse Cy3 (1:500; catalog \#115-165-146, lot 98592, The Jackson Laboratory).

Multiple electrode array recordings and analysis. Cultures were plated on MEA plates containing $120 \mathrm{TiN}$ recording and four internal reference electrodes (Multichannel Systems). Electrodes are $30 \mu \mathrm{m}$ in diameter and spaced $200 \mu \mathrm{m}$ apart. Data were acquired using an MEA2100Standard amplifier (Multichannel Systems) with a sampling rate of $10 \mathrm{kHz}$ per electrode. Recordings with MEA were previously shown to stay stable for $48 \mathrm{~h}$ under control conditions (Slomowitz et al., 2015). Recordings were conducted under constant $37^{\circ} \mathrm{C}$ and $5 \% \mathrm{CO}_{2}$ conditions, identical to incubator conditions. XE991 was added on the MEA plates for up to $48 \mathrm{~h}$. Control recordings were performed for $3 \mathrm{~h}$ before addition of the blocker. The baseline for analyses was defined as the mean of the three time points before XE991 addition. Percentage of the baseline was calculated and plotted for each hour during XE991 exposure. Spike sorting was performed offline on the recordings of the first 20 min from each hour, as it was shown previously that it represents well the full data (Slomowitz et al., 2015). In brief, the raw data were filtered offline at $200 \mathrm{~Hz}$ using a Butterworth high-pass filter. Spikes cutouts were then detected offline using MC Rack software (Multichannel Systems) based on a fixed threshold set to between 5 and 6 SDs from noise levels. Spike cutouts were then transferred to Offline Sorter (Plexon) for spike sorting. Spikes were plotted in 2-D or 3-D principal component (PC) space and unit clusters were semiautomatically detected using K-means clustering algorithm followed by template sorting. Clusters were then manually inspected to ensure stability throughout the experiment. Only clusters that fulfilled the following requirements were considered units and were used for analysis: (1) there was no spiking during the absolute refractory period; (2) the clusters were well defined relative to other clusters from the same electrode throughout the entire experiment; (3) there were no sudden jumps in cluster location on PC axes; and (4) the cluster is not centered on the origin of the PC axes. Analyses of MFR were performed using custom-written scripts in MATLAB (MathWorks). All the parameters were calculated by averaging the mean firing rates of all units for a given time point, per experiment. The eight experiments were then pooled, and the averages were plotted on the graphs. Unless otherwise noticed, we opted for this form of presentation, which gives equal weight to each repeat of the experiment. We nevertheless observed similar results when we pooled all the units without discerning between experiments. For MFR, similar results were observed with the unsorted data and after spike sorting.

Data analysis and statistics. For all experiments, data were collected from at least three different batches or more. Control cells were collected from each batch to minimize possible variations between batches. All graphs were built with Prism 8.0 (GraphPad Software). Error bars represent the SEM. In acute XE991 treatments that were conducted in the same neuron, statistical comparisons between untreated and treated cells were performed with two-tailed paired $t$ test. In chronic treatments that were conducted in two independent groups of cells, statistical comparisons were performed with two-tailed unpaired $t$ test. When treatments involved more than two independent groups of cells without matching between measures, statistical comparisons were performed with one-way ANOVA and post hoc Tukey's multiple-comparison test. For firing rateinput (F-I) curves, when treatments involved more than two independent groups of cells with matching between measures, data were analyzed with the Bonferroni multiple-comparison test, using SPSS. In acute XE991 treatments that were performed on the same cell, statistical comparisons were performed with a two-tailed paired test. In chronic treatments that were performed in two independent groups of cells, statistical comparisons were performed with a two-tailed unpaired test. Multiple comparisons were corrected with Bonferroni correction. Analyses of patch-clamp recordings were performed with Clampfit 10.4 (Molecular Devices). To analyze the afterdepolarization (ADP) size, we measured the ADP area. The ADP area was measured as the area under the signal confined between the lowest point separating the spike that precedes the 
ADP and the ADP itself until the return of the signal to RMP. For sEPSC and sIPSC analysis, the baseline of the traces was manually adjusted and the area under (for sEPSCs) or over (for sIPSCs) the baseline was defined as the charge transfer. For mEPSCs analysis, a template was created for each cell based on the shape of several mEPSCs merged together, and events were detected based on this template. The data taken to plot the cumulative probability of mEPSCs consists of 100 mEPSCs randomly selected for each cell and pooled for each condition. Images from immunostaining experiments were obtained using confocal microscopy (TCS SP5 microscope, Leica) with oil-immersion objectives of $63 \times$. Fields containing hippocampal neurons from primary cultures were indiscriminately selected. Quantifications were achieved with ImageJ (National Institutes of Health). Distances from the soma to the AIS were measured as described previously (Lezmy et al., 2017). For each experiment, the images were split into two groups, and the measurements were performed in a blinded manner independently by two different individuals. The results were compared before pooling the data together. Images were converted to TIFF files and imported into MATLAB for analysis, using a self-written algorithm. Semiautomatically, the algorithm recognizes the soma and the axon. At each pixel along the recognized axon, fluorescence intensity is measured. The AIS was defined to be between the proximal and distal points along the axons, in which the fluorescence intensity was $>50 \%$ of the maximum. The AIS length and distance from the soma were calculated and exported to a Microsoft Excel file.

\section{Results}

M-channel inhibition induces fast adaptive changes in intrinsic excitability of pyramidal excitatory neurons that depend on ongoing spiking activity

Recently, we have shown that sustained M-channel blockade by XE991 concomitantly triggers a fast ( $1-4 \mathrm{~h}$ ) adaptation of intrinsic excitability and a distal relocation of $\mathrm{K}_{\mathrm{v}} 7.3$ and $\mathrm{Na}_{\mathrm{v}}$ channels along the AIS in cultured hippocampal neurons and in CA1 pyramidal neurons of acute hippocampal slices (Lezmy et al., 2017). Here, we asked whether this fast homeostatic adaptation of intrinsic excitability depends on ongoing spiking activity. To address this question, we exposed hippocampal neurons to the M-channel blocker XE991 (10 $\mu \mathrm{M})$, in the presence of $1 \mu \mathrm{M}$ TTX, the $\mathrm{Na}^{+}$channel blocker, to suppress spontaneous firing for $4 \mathrm{~h}$, the time necessary for intrinsic homeostatic plasticity to occur. Whole-cell patch-clamp recordings from pyramidal-like excitatory neurons of hippocampal cultures (14-16 DIV) show that acute XE991 $(10 \mu \mathrm{M})$ exposure induced neuronal hyperexcitability, as reflected by a significant decrease in the threshold current ( $n=22$; control, $514 \pm 40 \mathrm{pA}$; acute XE991, $350 \pm 31 \mathrm{pA}$; oneway ANOVA, ${ }^{* *} p<0.000, F_{(4,112)}=14.65$; Fig. $\left.1 A, C\right)$ and a depolarized RMP ( $n=22$; control, $-60.2 \pm 1.0 \mathrm{mV}$; acute XE991, $-55.2 \pm 1.6 \mathrm{mV}$; Lezmy et al., 2017). Following $4 \mathrm{~h}$ incubation with XE991, the threshold current and the RMP returned to values similar to those of untreated neurons (Fig. $1 B, C$; one-way ANOVA, Sidak's multiple-comparisons test, control vs $4 \mathrm{~h}$ XE991, $p=0.4484$ ). This fast adaptation of intrinsic excitability was paralleled by a distal relocation of FGF14, a protein that physically bridges $\mathrm{Na}_{\mathrm{v}} 1.6$ and $\mathrm{K}_{\mathrm{v}} 7.2$ channels at the AIS (Pablo and Pitt, 2017). The distance between the soma and the proximal boundary of the FGF14-immunoreactive signal increased significantly and reached steady values after $4 \mathrm{~h}$ of XE991 exposure (untreated: $5.96 \pm 0.78 \mu \mathrm{m} ; n=69 ; 4 \mathrm{~h} \mathrm{XE991:} 12.16 \pm 0.79 \mu \mathrm{m}$; $n=95$; one-way ANOVA, $F_{(3,296)}=16.46, p<0.0001$; Fig. $\left.1 D, E\right)$. No changes in the total length of the FGF14 segment were observed (Fig. 1F). The extent of this distal redistribution remained the same following longer XE991 exposure for up to $2 \mathrm{~d}$ (Lezmy et al., 2017). However, when $1 \mu \mathrm{M}$ TTX was added together with $10 \mu \mathrm{M}$ XE991 for $4 \mathrm{~h}$ and washed out just before patch-clamp recording, it prevented the intrinsic homeostatic adaptations. Under these conditions, the acute effect of XE991 was preserved (Fig. 1B,C). Thus, 4 h of treatment with XE991 + TTX decreased significantly the threshold current $(339 \pm 14 \mathrm{pA}$, $n=26$; one-way ANOVA, Sidak's multiple-comparisons test, control vs 4 h XE991 + TTX $p=0.0002$ ) compared with 4 h exposure to XE991 alone $(578 \pm 24 \mathrm{pA}, n=30$; control vs $4 \mathrm{~h}$ XE991, $p=0.4484)$, TTX only ( $521 \pm 40 \mathrm{pA}, n=17$; control vs TTX, $p=0.9126$ ), or control neurons ( $514 \pm 40 \mathrm{pA}, n=22$; Fig. 1C). Similarly, TTX prevented the distal relocation of FGF14 along the AIS (Fig. 1D,E; one-way ANOVA, Sidak's multiple comparisons, untreated vs $4 \mathrm{~h} \mathrm{XE991}+$ TTX, $p=0.8589)$. This result indicates that the intrinsic homeostatic plasticity achieved within $4 \mathrm{~h}$ is clearly contingent on ongoing spiking activity.

\section{M-channel blockade triggers homeostasis of spike afterdepolarization}

M-channels are known to control spike ADP and burst generation in hippocampal CA1 pyramidal neurons (Yue and Yaari, 2004). The inhibition of M-currents allows the spike ADP to grow, which can convert solitary spikes into bursts of action potentials (Chen and Yaari, 2008); therefore, large ADPs cause neurons to fire in bursting mode. Here, in CA1 pyramidal neurons from hippocampal slices, acute M-channel inhibition by XE991 $(30 \mu \mathrm{M})$ promoted an increase in the spike ADP size (ADP area increased from $582 \pm 135$ to $1223 \pm 368 \mathrm{mV} / \mathrm{ms}$; $n=6$; paired two-tailed $t$ test, $p=0.047$; Fig. $2 A-C$ ). The increase in ADP size generated another spike in $50 \%$ of the cells. In these neurons, the increase in burst firing on acute XE991 exposure was nullified following sustained M-channel inhibition, in conjunction with a decrease in ADP size, which returned to the baseline values within $3 \mathrm{~h}$ of XE991 treatment (ADP area, $380 \pm$ $129 \mathrm{mV} / \mathrm{ms} ; n=6$; Fig. $2 A-C)$. In the other neurons, the increase in ADP size was not enough to induce an additional spike, but the initial increase in ADP size was still followed by a decrease to the baseline ADP size after prolonged XE991 treatment (Fig. 2B). These results suggest that the fast intrinsic homeostatic adaptation triggered by sustained M-channel blockade includes the size of the ADP, which is meaningful for the bursting firing pattern.

\section{M-channel block triggers a slow compensation of the mean firing rate}

Next, we examined how sustained M-channel inhibition affects the spontaneous ongoing firing rate of hippocampal neurons. Under this experimental setting, we previously showed that Mcurrent blockage by $10 \mu \mathrm{M}$ XE-991 was maintained at the same level ( $\sim 70 \%$ inhibition) when neurons were chronically exposed to the drug for extended periods of 1-48 h (Lezmy et al., 2017). A significant increase in the ongoing firing rate was found after acute M-channel inhibition ( $\sim 1.6$-fold increase; two tailed paired $t$ test, $p=0.0012, n=11)$ and was still observed following $1-4$ h of XE991 chronic treatment $(\sim 1.5$-fold increase; one-way ANOVA, $p=0.0057 ; n=26-84$; Fig. $3 A-C$ ). At longer XE991 exposure times ( 24 and $48 \mathrm{~h}$ ), the ongoing firing rate progressively decreased and returned to the values of untreated neurons (Fig. $3 C$ ), reflecting an additional slow homeostatic plasticity process.

To test the time course of homeostatic compensation of the MFR to chronic M-channel blockade at the level of neuronal population, we tested the effect of XE991 on spontaneous firing properties of cultured hippocampal neurons grown on an MEA for $\sim 2-3$ weeks. Each MEA contains 120 recording electrodes, whereas each electrode is capable of recording the activity of several adjacent neurons (Slomowitz et al., 2015; Styr et al., 2019). 
A

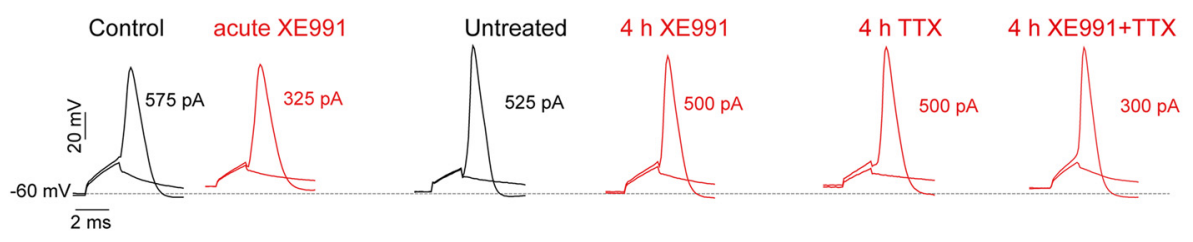

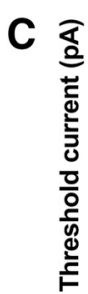

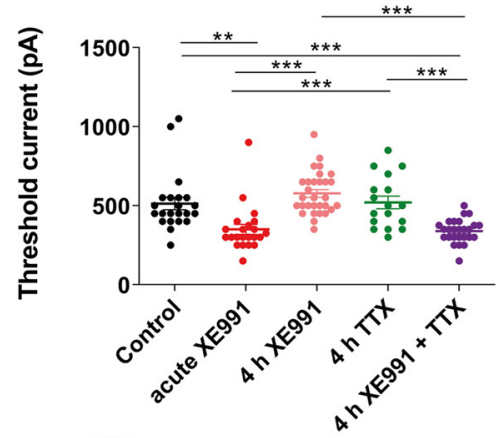

D

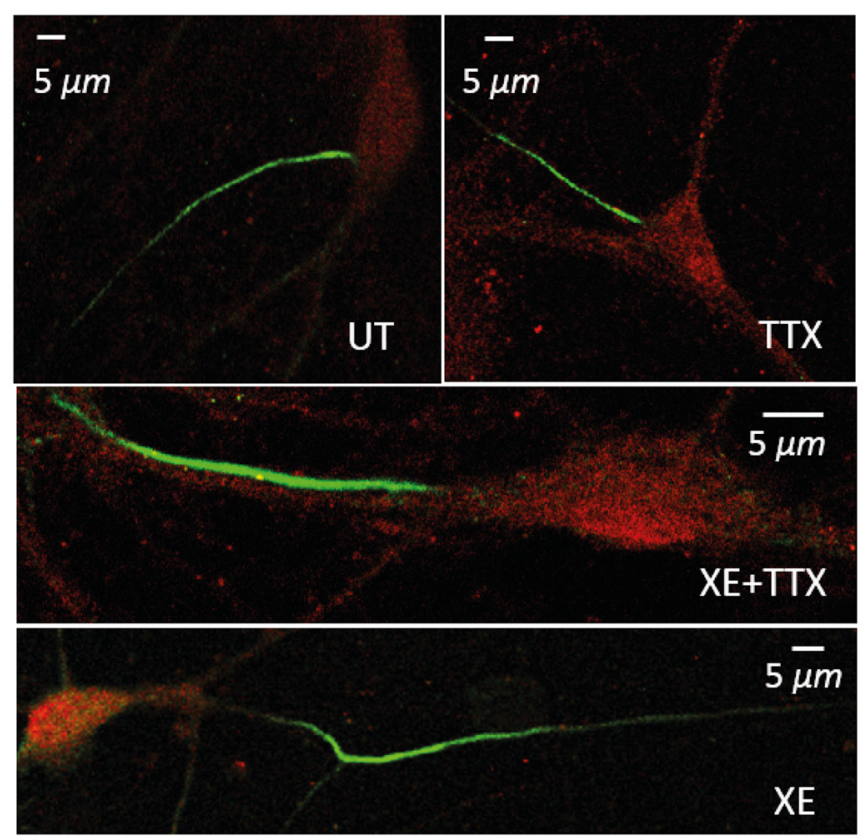

E

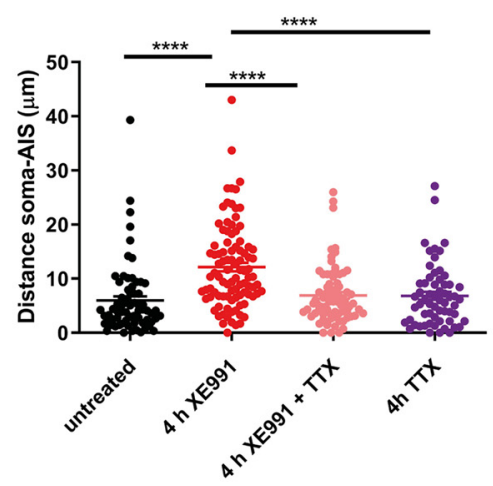

$\mathbf{F}$

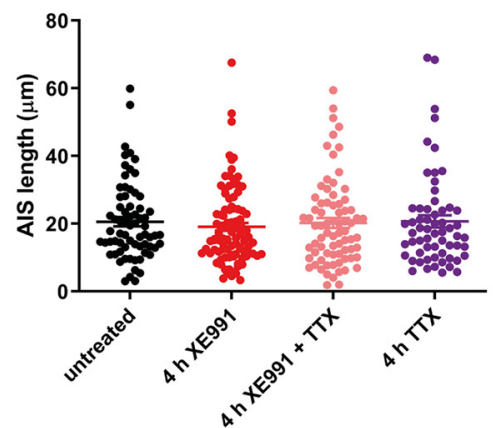

Figure 1. M-channel inhibition triggers fast adaptive changes in intrinsic excitability and AIS FGF14 distal relocation that tightly depend on ongoing spiking activity. $\boldsymbol{A}, \boldsymbol{B}$, Representative traces of solitary spike discharge evoked by $2 \mathrm{~ms}$ step injection of depolarizing currents with increments of $25 \mathrm{pA}$ : in control conditions (black) and following acute XE991 exposure (red; $\boldsymbol{A}$ ); and in control untreated conditions (black) and following $4 \mathrm{~h}$ exposure to XE991, TTX, and TTX + XE991 (red; $\boldsymbol{B}$ ). In each case, the values of the threshold current are shown. $\boldsymbol{C}$, Acute XE991 exposure significantly decreased the threshold current $\left(n=22\right.$; control, $514 \pm 40 \mathrm{pA}$; acute XE991, $350 \pm 31 \mathrm{pA}$; one-way ANOVA, $\left.F_{(4,112)}=14.65,{ }^{* *} p=0.002,{ }^{* * *} p<0.0001\right)$. The threshold currents of neurons exposed to $4 \mathrm{~h} \mathrm{XE991} \mathrm{(} n=30,578 \pm 24 \mathrm{pA})$ or to $4 \mathrm{~h} \mathrm{TTX} \mathrm{(} n=17,521 \pm 40 \mathrm{pA})$ were not significantly different from control (one-way ANOVA, Sidak's multiple-comparisons test; control vs $4 \mathrm{~h}$ XE991, $p=0.4484$; and control vs $4 \mathrm{~h} \mathrm{TTX}, p>0.9999)$. In contrast, treatment with XE991 + TTX for $4 \mathrm{~h}(n=26,339 \pm 14 \mathrm{pA})$ significantly decreased the threshold current compared with that of TTX alone or to control (one-way ANOVA, control vs $4 \mathrm{~h} \mathrm{XE991}+\mathrm{TTX}, p=0.0002$ ). D, Representative FGF14 (green) and MAP2 (red) immunostaining label the AIS and the somatodendritic compartment, respectively. The AIS relocated distally away from the soma following $4 \mathrm{~h}$ of exposure to XE991, but TTX prevents this effect. $\boldsymbol{E}$, The FGF14-immunoreactive signal relocated away from soma following $4 \mathrm{~h}$ of exposure to XE991 (untreated: $5.96 \pm 0.78 \mu \mathrm{m} ; n=69 ; 4 \mathrm{~h}$ XE991: $12.16 \pm 0.79 \mu \mathrm{m} ; n=95 ;$ one-way ANOVA, $F_{(3,296)}=16.46$, $*^{* * *} p<0.0001$ ). Addition of TTX together with XE991 prevented the distal shift of the AIS. $\boldsymbol{F}$, No significant changes in the total length of the FGF14 segment were observed (one-way ANOVA, $\left.F_{(3,298)}=0.3143, p=0.8151\right)$

Under control conditions, the average firing rates of the hippocampal network showed long-term stability for up to $48 \mathrm{~h}$ (Fig. $3 F$; Slomowitz et al., 2015). Spiking network activity was continuously monitored in an incubator chamber during a baseline recording period and for $2 \mathrm{~d}$ following application of $10 \mu \mathrm{M}$ XE991. XE991 application caused an immediate 2.14-fold increase in

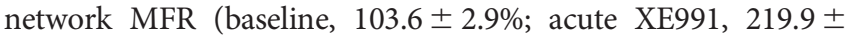
33.1\%; one-way ANOVA, Friedman test with Dunn's multiplecomparisons test, $p<0.0002$ ), which remained elevated $2 \mathrm{~h}$ after XE991 application ( $2 \mathrm{~h}$ XE991: $172.3 \pm 17.8 \%, p<0.0021)$. Then, MFR gradually returned to the baseline values (140.0 \pm $13.5 \%$ and $103.5 \pm 13.7 \%, 1$ and $2 \mathrm{~d}$ after XE991 application, respectively; Fig. $3 D, E)$. These results demonstrate that, similar to other chronic pharmacological perturbations inducing network hyperactivity, such as $\mathrm{GABA}_{\mathrm{A}}$ receptor blockade (Vertkin et al., 2015) and glutamate uptake blockade (Styr et al., 2019), the recovery of mean firing rates following M-type potassium channel blockade occurs at a slow timescale of $1-2 \mathrm{~d}$.

\section{Chronic M-channel inhibition triggers a slow homeostasis of E/I ratio}

To examine the potential alterations in the synaptic output of the network, sEPSCs and sIPSCs were recorded from pyramidal-like excitatory hippocampal neurons by holding the cells successively at the inhibitory and excitatory reversal potentials, respectively (see Materials and Methods). Acute XE991 treatment promoted a significant increase in the sEPSCs charge transfer ( $n=17$; control, $25.0 \pm 4.3 \mathrm{pA} / \mathrm{s}$; acute XE991, $43.5 \pm 9.5 \mathrm{pA} / \mathrm{s}$; two-tailed paired $t$ test, ${ }^{\star} p=0.043$; Fig. $4 A, B$ ) but did not have a significant effect on sIPSCs (Fig. 4C,D). Therefore, the E/I ratio was 
A

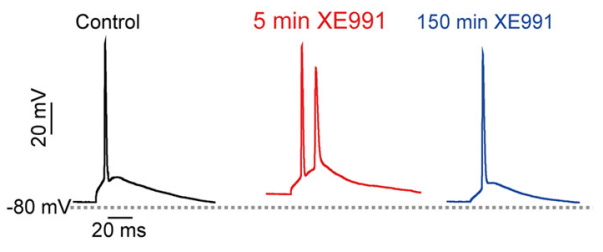

B

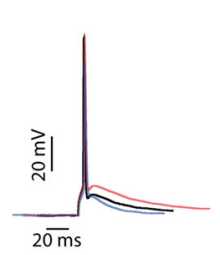

C

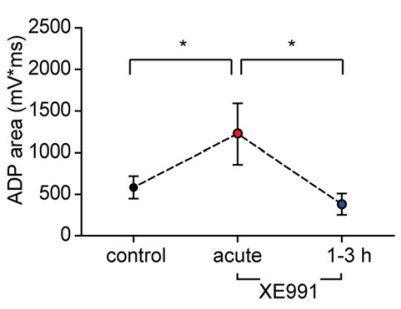

Figure 2. Afterdepolarization size readjust faster than the mean firing rate. $\boldsymbol{A}$, Representative traces of spike discharges evoked by 5 ms step injection of depolarizing threshold currents in the same CA1 neuron from hippocampal slice before (black), after 5 min (red), and after 150 min (blue) of XE991 exposure. In this neuron, acute XE991 treatment increased the ADP size and generated a doublet, which was reversed to a single spike following extended XE991 treatment. $\boldsymbol{B}$, Representative traces of spike discharges evoked by 5 ms step injection of depolarizing threshold currents in a CA1 neuron in which the ADP size changed without leading to burst generation. The traces were superimposed, and the RMPs were aligned to emphasize the initial increase and the adaptation in the ADP size throughout XE991 exposure. C, Graph gathering the data from six different cells. Acute XE991 treatment significantly increased the ADP size (control, $582 \pm 135 \mathrm{mV} / \mathrm{ms}$; acute, $1223 \pm 368 \mathrm{mV} / \mathrm{ms}$; two-tailed paired $t$ test, vs acute: ${ }^{*} p=0.0474, n=6, t=2.614$, df $\left.=5\right)$ and decreased it following prolonged exposure $(1-3 \mathrm{~h}$ : $380 \pm 129 \mathrm{mV} / \mathrm{ms}$; two-tailed paired $t$ test, vs $1-3 \mathrm{~h}:{ }^{*} p=0.0218, n=6, t=3.288, \mathrm{df}=5$ ).

significantly increased by acute XE991 exposure $(n=17$; control, $0.35 \pm 0.06$; acute XE991, $0.77 \pm 0.15$; two-tailed paired $t$ test, ${ }^{\star} p=0.0214$; Fig. $4 E$ ). After $4 \mathrm{~h}$ of M-channel inhibition, the sEPSC charge transfer was still higher than that of untreated neurons; then, following 24 and $48 \mathrm{~h}$ of XE991 exposure, the sEPSC charge transfer returned to values similar to those of untreated neurons [Fig. $4 B$; one-way ANOVA: ${ }^{*} p=0.03, F_{(3,193)}=3.044$; post hoc Tukey's multiple-comparison test: significantly different untreated $(n=68)$ vs 4 h XE991 $(n=50)]$. In contrast, the sIPSC charge transfer values after 4,24 , and 48 h of XE991 treatment were similar to those of the control neurons (Fig. 4D). Thus, after $4 \mathrm{~h}$ of M-channel blockade we still observed an increase in the E/ I ratio, similar to that of the acute XE991 exposure (untreated: $0.53 \pm 0.04, n=68 ; 4$ h XE991: $0.74 \pm 0.1, n=50)$. Following 24 $\mathrm{h}(n=45)$ and $48 \mathrm{~h}(n=34)$ of XE991 treatment, the E/I ratio returned back to initial values of untreated neurons (Fig. $4 E$ ).

\section{Chronic M-channel inhibition triggers a slow homeostatic scaling}

To understand the mechanisms by which the MFR and the sEPSC charge transfer slowly compensate following chronic Mchannel inhibition, we examined the mEPSCs properties. The frequency and amplitude of mEPSCs were not modified by acute XE991 exposure (Fig. 5A,C). Prolonged treatment with XE991 $(4,24,48 \mathrm{~h})$ did not significantly alter the frequency of mEPSCs (Fig. $5 B$ ). In contrast, the amplitude of mEPSCs significantly decreased following $48 \mathrm{~h}$ of M-channel block (Fig. 5D,E; $n=49-$ 68 ; one-way ANOVA: $p=0.0117, F_{(3,219)}=3.752$; Tukey's multiple-comparisons test, untreated vs $48 \mathrm{~h}$ XE991, significantly different), suggesting that slow modifications in excitatory transmission occur on a timescale of days.

\section{Lack of homeostatic plasticity at the level of intrinsic} excitability and spontaneous MFR in GABAergic inhibitory neurons following chronic XE991 treatment

So far, our results indicate that the fast $(\sim 4 \mathrm{~h})$ intrinsic homeostatic plasticity of excitatory neurons is not sufficient for the homeostatic normalization of the MFR, a slow process that needs $2 \mathrm{~d}$ to reach the baseline values. To explore the mechanisms underlying this mismatch in timescales, we set out to examine the intrinsic excitability and the spontaneous mean firing rate of GABAergic inhibitory neurons in the same hippocampal cultures following acute and extended exposure to the M-channel blocker XE991 (10 $\mu \mathrm{M})$. To identify GABAergic neurons, we infected hippocampal cultures with a recombinant virus derived from an $\mathrm{AAV}$ vector driving the expression of the fluorescent protein
mCherry under the control of the specific GABAergic hDlx promoter (Fig. 6A; Dimidschstein et al., 2016). The Dlx promoter was shown to restrict reporter expression in vivo to all GABAergic interneurons in the forebrain, including hippocampus, as well as in cultured neurons in vitro (Dimidschstein et al., 2016). Based on this method, we estimate that GABAergic inhibitory neurons represent $\sim 20 \%$ of the total neuronal population in our cultures. Whole-cell patch-clamp recordings from GABAergic neurons show that acute XE991 $(10 \mu \mathrm{M})$ exposure induced hyperexcitability, as reflected by a significant decrease in the threshold current (Fig. $6 B, C ; n=27$; control, $480 \pm 20 \mathrm{pA}$; acute XE991, $369 \pm$ $17 \mathrm{pA}$; one-way ANOVA, Tukey's multiple-comparisons test, $\left.p=0.0013, F_{(2,69)}=7.324\right)$. Following $4 \mathrm{~h}$ incubation with XE991, the threshold current was not significantly different from that of the acute treatment (Fig. $6 B, C ; n=18 ; 4 \mathrm{~h}$ XE991; $431 \pm 31 \mathrm{pA}$; one-way ANOVA, $p=0.1441$ ). Although the threshold current increased, it did not return to the values of untreated neurons. F-I curve experiments in GABAergic neurons confirmed this trend where spike discharges were evoked by incremental depolarizing current injections for $400 \mathrm{~ms}$ (Fig. 6D,E). Acute XE991 (10 $\mu \mathrm{M})$ exposure significantly increased the frequency of evoked spike discharge $(n=26$; Bonferroni multiple-comparison test, control vs acute; $250 \mathrm{pA}:{ }^{* *} p=0.0036, t=3.209, \mathrm{df}=25 ; 300 \mathrm{pA}:{ }^{* *} p=0.0042$, $t=3.151, \mathrm{df}=25 ; 350 \mathrm{pA}:{ }^{\star} p=0.0168, t=2.562, \mathrm{df}=25 ; 400 \mathrm{pA}:$ $\left.{ }^{\star} p=0.0191, t=2.549, \mathrm{df}=20\right)$. However, following $4 \mathrm{~h}$ incubation with XE991, the F-I curve still showed increased frequency of spike discharge and was not significantly different from acute XE991 treatment (Fig. 6D,E; $n=20-26$, two-tailed unpaired $t$ test, where multiple comparisons were adjusted with multiple $t$ tests and Bonferroni correction). For comparison, in pyramidal-like excitatory neurons, acute XE991 exposure significantly increased the frequency of evoked spike discharge but sustained the block of M-channels (4 $\mathrm{h}$ XE991) led to rapid adaptive changes that brought the values back to those of untreated neurons (Fig. 6F,G; $n=47-68$; Bonferroni multiple-comparison test, control vs acute: $50 \mathrm{pA}:{ }^{\star} p=0.0137, t=2.985, \mathrm{df}=10 ; 100 \mathrm{pA}:{ }^{\star} p=0.0166, t=2.863$, $\mathrm{df}=10 ; 150 \mathrm{pA}:{ }^{*} p=0.0074, t=3.348, \mathrm{df}=10$; control vs $4 \mathrm{~h}$ XE991: 50 pA: $p=0.3988, t=0.8470, \mathrm{df}=113 ; 100 \mathrm{pA}: p=0.9400$, $t=0.07,548, \quad \mathrm{df}=113 ; 150 \mathrm{pA}: p=0.6537, t=0.4498, \mathrm{df}=112$; $200 \mathrm{pA}: p=0.5964, t=0.5311, \mathrm{df}=113 ; 250 \mathrm{pA}: p=0.5722, t=$ $0.5665, \mathrm{df}=113$ ). Along the same line, the lack of fast intrinsic adaptation of GABAergic neurons was paralleled by the lack of a distal relocation of FGF14. Immunocytochemical colabeling of GABAergic inhibitory neurons by vGAT and FGF14 indicates that following $4 \mathrm{~h}$ of XE991 treatment no significant changes were observed in the distance between the soma and the proximal 
A

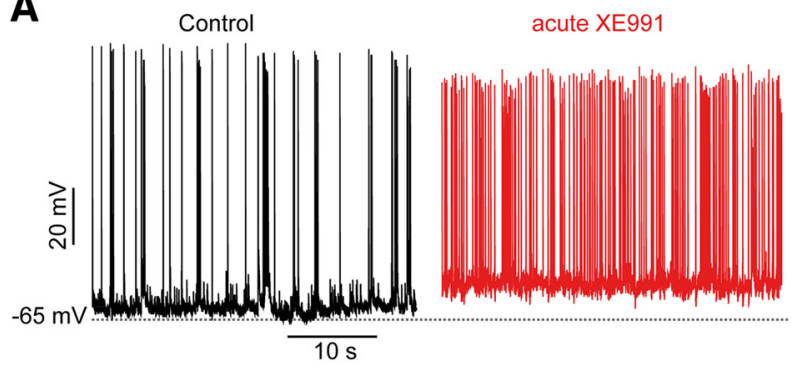

B

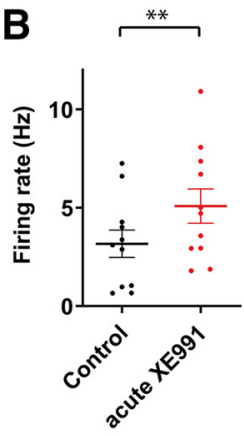

C

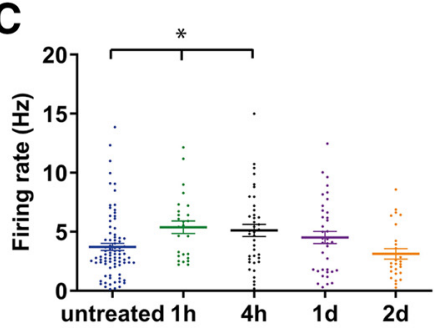

D

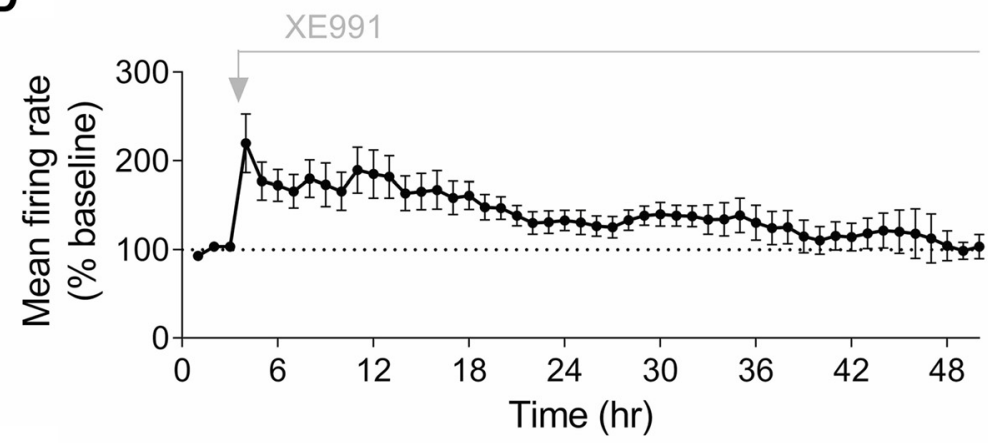

$\mathbf{F}$

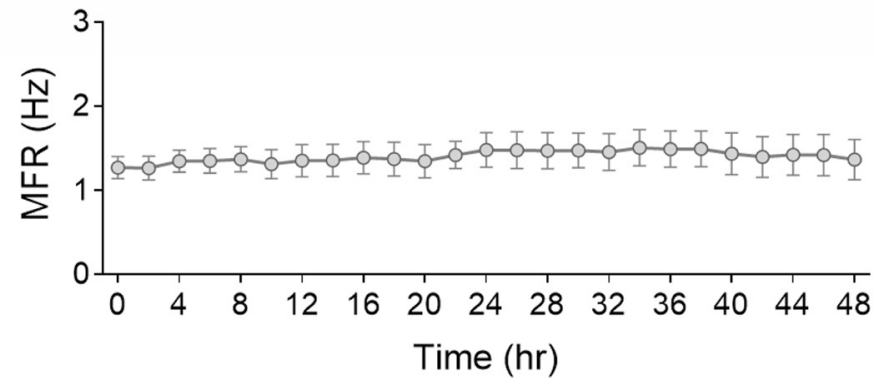

E

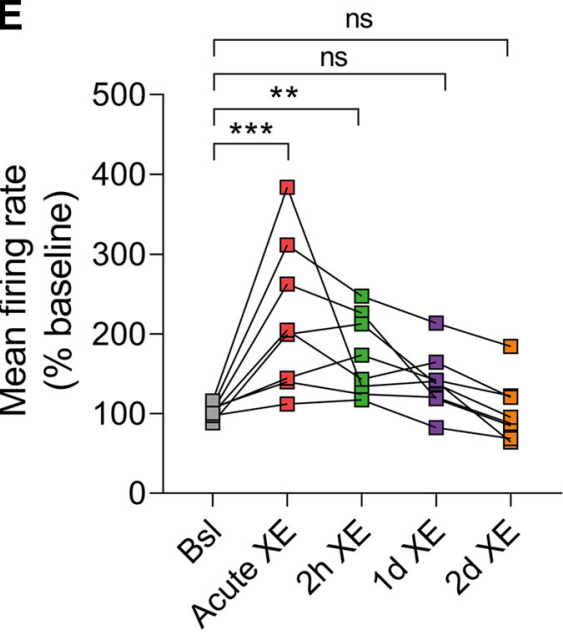

Figure 3. The fast homeostatic adaptation in intrinsic excitability is followed by a slow compensation of the mean firing rate. $\boldsymbol{A}$, Representative traces showing the larger spontaneous firing rate after acute XE991 treatment (red trace) compared with control (Black trace). B, Acute XE991 exposure significantly increased the ongoing firing rate by 1.7-fold (two-tailed paired $t$ test: ${ }^{* *} p=0.0012, n=11, t=4.495, \mathrm{df}=10$ ). $C$, With longer exposures to XE991, the spontaneous firing rate progressively decreased and returned to values close to those of untreated neurons [one-way ANOVA: $F_{(4,207)}=3.749,{ }^{* *} p=0.0057$; and Dunnett's multiple-comparisons test: significantly different for untreated $(n=84)$ vs $1 \mathrm{~h}(n=21)$ and $4 \mathrm{~h}(n=39)$, but not significant vs $24 \mathrm{~h}(n=37)$ and $48 \mathrm{~h}(n=26)]$. $D$, Analysis of the MFR at the level of network across eight MEA experiments ( 238 single units) over the course of $2 \mathrm{~d}$ of recording in the presence of $10 \mu \mathrm{m}$ XE991. Each recording consisted of $3 \mathrm{~h}$ of baseline and $48 \mathrm{~h}$ following XE991 administration. $\boldsymbol{E}$, Statistical comparison of the time points shown (the same data as in $\boldsymbol{D}$ ). Significant increase in MFR following acute and $2 \mathrm{~h}$ of XE991 (219.9 $\pm 33.1 \%$ and $172.3 \pm 17.8 \%$, respectively) and MFR renormalization following 1 and $2 \mathrm{~d}(140.0 \pm 13.5 \%$ and $103.5 \pm 13.7 \%$, respectively). One-way ANOVA, Friedman test with Dunn's multiple-comparisons test: ${ }^{*} p<0.05,{ }^{* *} p<0.0021,{ }^{* * *} p<0.0002$; ns, not significant). $\boldsymbol{F}$, Mean firing rate of $48 \mathrm{~h}$ control MEA recordings [average of six experiments (440 single units)]. Error bars represent the SEM.

boundary of the FGF14-immunoreactive signal and in the total length of the FGF14 segment (Fig. 6H-J; $n=90-93$; unpaired twotailed $t$ test: $p=0.7632, t=0.3017, \mathrm{df}=181$; and $p=0.2049$, $t=1.272, \mathrm{df}=181$ ).

Next, we investigated the spontaneous MFR of GABAergic inhibitory neurons in the same hippocampal cultures following acute and $48 \mathrm{~h}$ exposure to the M-channel blocker XE991 (10 $\mu \mathrm{M})$. A significant increase in the ongoing firing rate was found after acute M-channel inhibition, which was still observed following $48 \mathrm{~h}$ of XE991 chronic treatment (Fig. $7 A-C$; $n=24$; control, $1.86 \pm 0.35 \mathrm{~Hz}$; acute XE991, $3.72 \pm 0.41 \mathrm{~Hz}$; one-way ANOVA and Tukey's multiple-comparisons test: $p=0.0048$, $F_{(2,72)}=5.754$; control vs acute, $p=0.0122$; control vs $48 \mathrm{~h}$ $\mathrm{XE991,} p=0.0122$ ). Together, these results indicate that in sharp contrast to pyramidal excitatory neurons, GABAergic neurons remained hyperexcitable due to the lack of homeostatic adaptation to M-channel blockade at the level of intrinsic excitability and spontaneous MFR (Figs. 6, 7).

\section{Discussion}

In neural networks, spontaneous firing arises from the complex interplay between synaptic currents and intrinsic neuronal properties. Homeostasis is essential for normal brain function as it can prevent deleterious states of epileptic activity or quiescence by providing negative feedback control. Many lines of evidence indicate that neuronal networks use various types of regulatory mechanisms to achieve homeostasis over a wide range of temporal and spatial scales (Turrigiano and Nelson, 2004; Davis, 2006; Marder and Goaillard, 2006; Turrigiano, 2011, 2017; Styr and Slutsky, 2018). Homeostatic synaptic scaling and homeostatic modulation of intrinsic excitability regulate firing rates; however, it is not clear whether these two forms of homeostasis are redundant or induced in parallel to fulfill distinct functions, or whether these two processes are interacting to achieve complementary tasks.

In the present work, homeostatic plasticity was triggered by chronic M-channel blockade in hippocampal cultures resulting 
A

Control Acute XE991

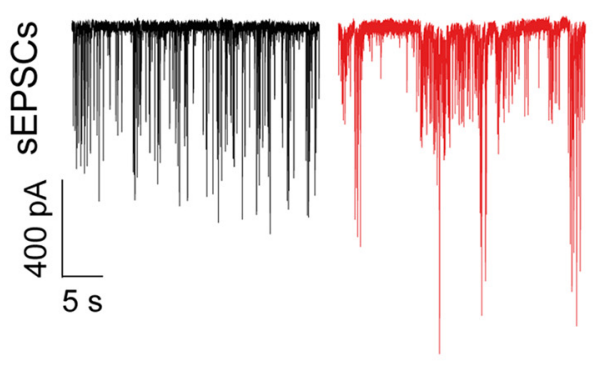

C

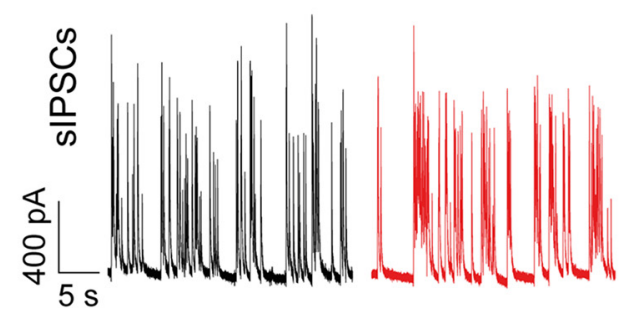

B

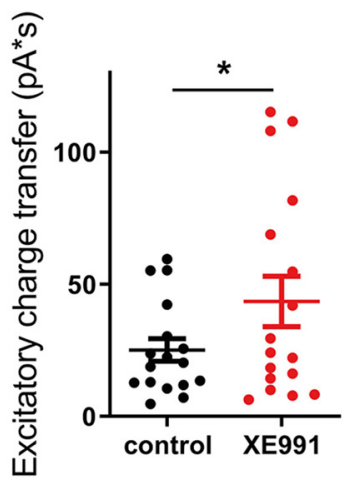

D

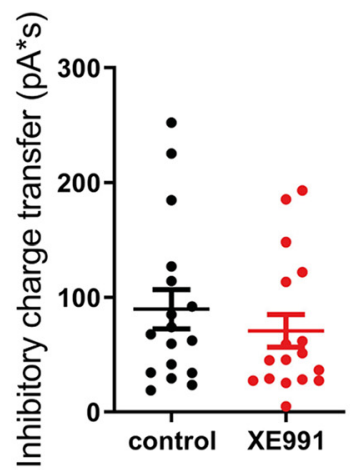

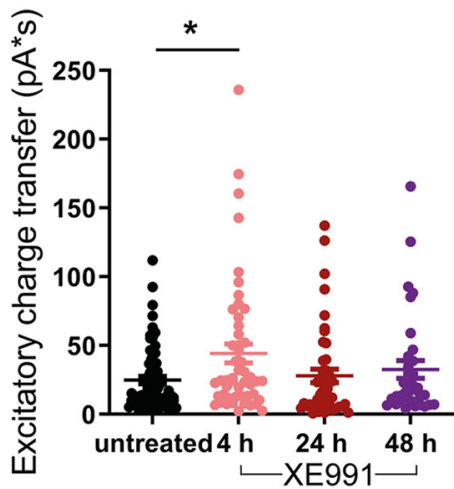

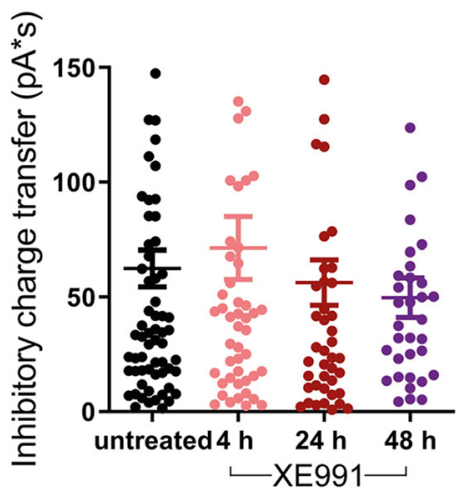

$\mathbf{E}$

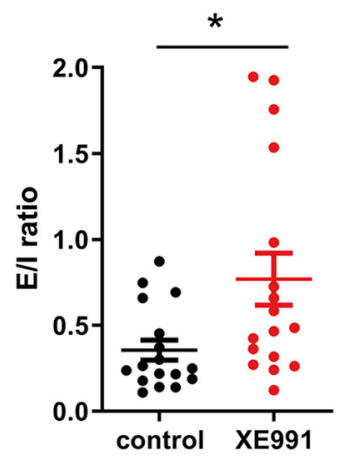

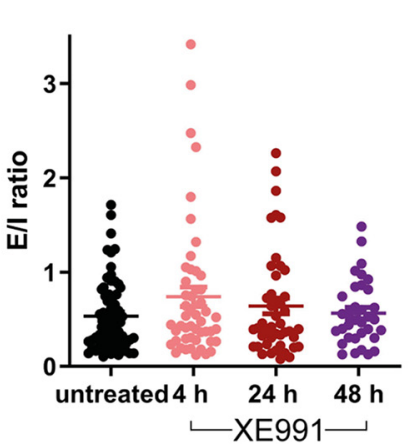

Figure 4. Chronic M-channel inhibition triggers a slow adaptation of E/I balance caused by changes in sEPSC charge transfer. $A$, Representative traces showing larger sEPSCs following acute XE991 exposure (red) compared with control (black). $B$, Acute XE991 exposure significantly increased the sEPSC charge transfer ( $n=17$; control, $25.1 \pm 4.3 \mathrm{pA} / \mathrm{s}$; acute XE991, $43.5 \pm 9.5 \mathrm{pA} / \mathrm{s}$; two-tailed paired $t$ test: ${ }^{*} p=0.043, t=2.198, \mathrm{df}=16$ ). After $4 \mathrm{~h}$ of XE991 treatment, the $\mathrm{SEPSC}$ charge transfer was still larger than that of the control, and it returned to values similar to baseline after extended treatments of $24 \mathrm{~h}(n=45)$ and $48 \mathrm{~h}\left[n=34\right.$; one-way ANOVA: ${ }^{*} p=0.03, F_{(3,193)}=3.044$; post hoc Tukey's multiple-comparison test: significantly different for untreated ( $n=68)$ vs $4 \mathrm{~h}(n=50)]$. C, Representative traces of sIPSCS before (black) and following acute XE991 exposure (red). $\boldsymbol{D}$, Acute (two-tailed paired $t$ test: $p=0.0763, t=1.895$, $\mathrm{df}=16$ ) and prolonged XE991 exposures (one-way ANOVA: $p=0.5651, F=0.6803, \mathrm{df}=3$ ) did not significantly change the sIPSC charge transfer. $E, E / I$ ratio was calculated by dividing sEPSC by sIPSC charge transfers of the same neurons. Acute XE991 significantly increased the E/l ratio (control, $0.35 \pm 0.06$; acute XE991, 0.77 \pm 0.15 ; ; two-tailed paired $t$ test: ${ }^{*} p=0.0214$, $t=2.549, \mathrm{df}=16)$. E/I ratio following prolonged XE991 treatments reflected the changes observed on the sEPSC charge transfer. The E/I ratio increased following $4 \mathrm{~h}$ of XE991 exposure, while it returned progressively to baseline value after 24 and $48 \mathrm{~h}$ of XE991 treatment (one-way ANOVA: $p=0.1835, F_{(3,193)}=1.631 ; n=45-68$ ).

in both intrinsic and synaptic homeostatic adaptations of pyramidal excitatory neurons. Acute $\mathrm{M}$-channel inhibition increases neuronal excitability and causes hippocampal CA1 pyramidal neurons to increase the spike ADP (Brown and Adams, 1980; Halliwell and Adams, 1982; Jentsch, 2000; Shah et al., 2002). Assembled as heterotetramers of $K_{v} 7.2$ and $\mathrm{K}_{\mathrm{v}} 7.3$ subunits, $\mathrm{M}$-channels generate subthreshold, noninactivating voltage-gated $\mathrm{K}^{+}$currents that play an important role in controlling neuronal excitability (Jentsch, 2000; Shah et al., 2002; Delmas and Brown, 2005). We found that a few hours of M-current blockade led to a progressive decrease in intrinsic excitability of hippocampal excitatory neurons
(Lezmy et al., 2017). This fast homeostatic adaptation was associated with a distal shift of the spike trigger zone and a distal relocation of FGF14, $\mathrm{Na}^{+}$, and $\mathrm{K}_{\mathrm{v}} 7$ channels along the AIS (Lezmy et al., 2017). Here, we showed that the fast (1-4 h) homeostatic intrinsic adaptation preceded a slow $(\sim 2 \mathrm{~d})$ homeostatic process of synaptic downscaling contributing to the compensation of the increased MFR. This was reflected by a decrease in mEPSC amplitude. Importantly, TTX coapplication with the M-channel blocker for $4 \mathrm{~h}$ was able to prevent intrinsic homeostatic plasticity by precluding the AIS FGF14 distal relocation and by restoring the values of the threshold current, similar to those of neurons acutely exposed 

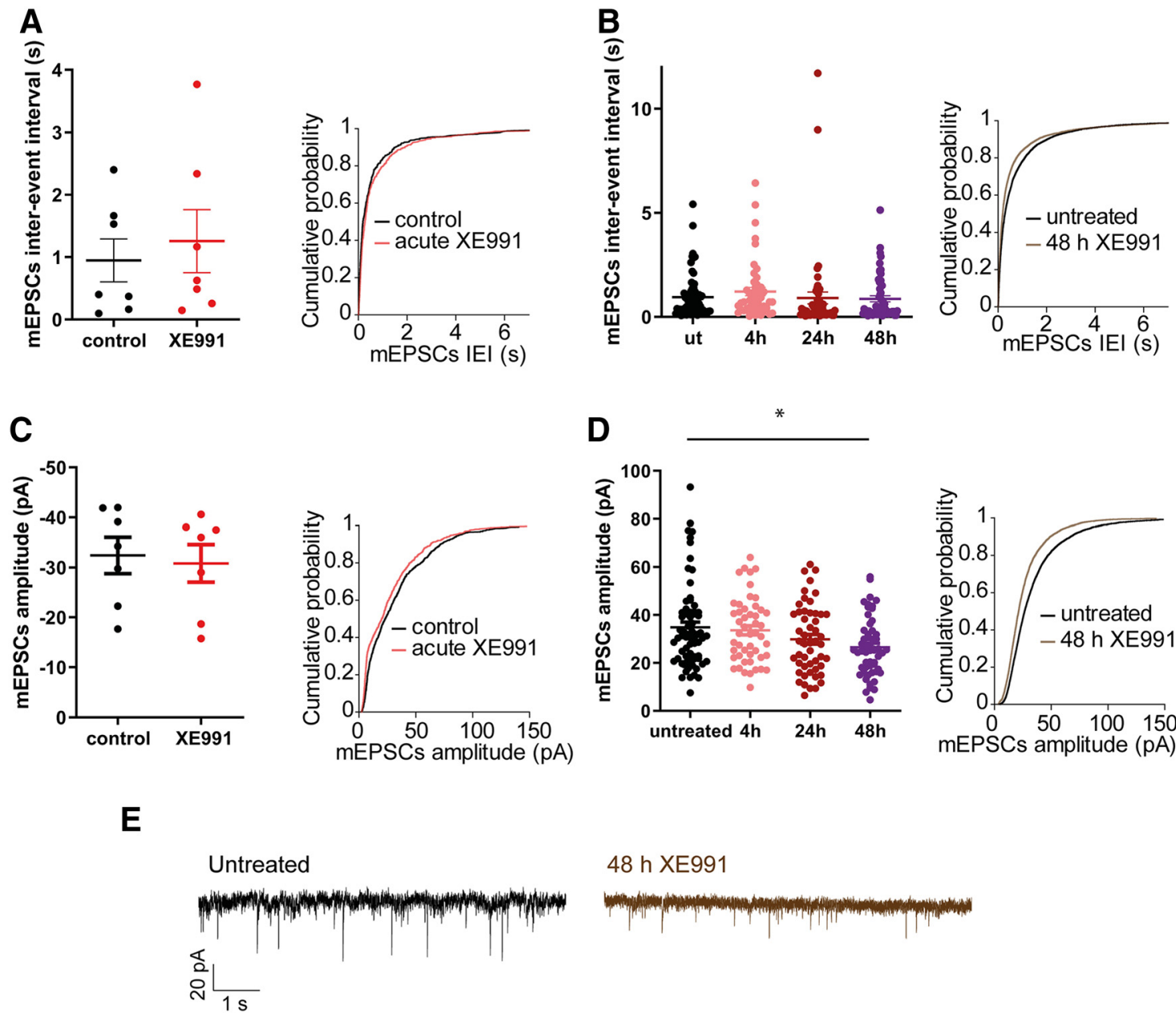

$48 \mathrm{~h}$ XE991

Figure 5. Chronic M-channel blockage promotes slow postsynaptic modulations. $\boldsymbol{A}-\boldsymbol{D}$, Bar charts and cumulative probability plots are shown for the same data in each panel. $\boldsymbol{A}, \boldsymbol{B}$, The distribution of mEPSC interevent intervals was not significantly different throughout acute $(n=7$, two-tailed paired $t$ test: $p=0.3903, t=0.9257$, df $=6)$ and extended XE991 exposures (oneway ANOVA: $\left.p=0.5963, F_{(3,219)}=0.6301, n=49-68\right)$. C, $\boldsymbol{D}$, The distribution of mEPSC amplitudes was similar during acute M-channel blockade (two-tailed paired $t$ test: $p=0.1419$, $t=1.691, \mathrm{df}=6)$ but was significantly reduced during exposure to XE991 for $48 \mathrm{~h}\left(n=49-68\right.$; one-way ANOVA: ${ }^{*} p=0.0117, F_{(3,219)}=3.752$; post hoc Tukey's multiple-comparison test: significantly different untreated vs $48 \mathrm{~h}$ ). $\boldsymbol{E}$, Representative traces showing the decrease in mESPC amplitude following $48 \mathrm{~h}$ of XE991 treatment.

to XE991. Thus, ongoing spiking activity is required for the fast adaptive change in intrinsic excitability of hippocampal excitatory neurons.

Concomitant to this process, extended M-channel blockade with XE991 triggered a fast adaptation of the spike ADP. In CA1 pyramidal cells, the persistent $\mathrm{Na}^{+}$current (INaP; French et al., 1990) in the perisomatic region is the predominant inward current generating the spike ADP (Azouz et al., 1996; Su et al., 2001; Yue et al., 2005; Golomb et al., 2006). The depolarizing action of $\mathrm{INaP}$ is mainly counteracted by M-channels. The M-current prevents the escalation of the spike ADP into a spike burst (Yue and Yaari, 2004, 2006). Here in CA1 pyramidal neurons of acute hippocampal slices, we showed that the XE991-induced increase in spike ADP size and the trend to fire in a burst adapted within $3 \mathrm{~h}$ of M-channel block and returned to the values of untreated neurons. Our data indicate that the adaptation of the spike ADP (2$3 \mathrm{~h}$ ) occurred concomitantly with the fast homeostatic changes in intrinsic excitability (but see Lezmy et al., 2017). The fast timescale of homeostatic intrinsic plasticity described in the present study significantly differs from that observed in cortical neurons following activity deprivation (TTX), which takes a longer time (24-48 h) to be induced (Desai et al., 1999; Bulow et al., 2019). Although we cannot exclude changes in ion channel conductances, these differences suggest that, depending on the nature of the plasticity trigger, different signaling pathways can be mobilized to produce multiple forms of intrinsic plasticity. In addition, we showed that the ongoing spiking potently affects the adaptive changes in intrinsic excitability. In light of our present data, we speculate that the transient rise in spiking activity of hippocampal excitatory neurons following $\mathrm{M}$-channel block may trigger the fast adaptive changes in intrinsic excitability associated with the distal shift of the spike trigger zone and the distal relocation of $\mathrm{Na}^{+}$and $\mathrm{K}_{\mathrm{v}} 7$ channels along the AIS (Lezmy et al., 2017). In support of this suggestion, a previous study showed that optogenetic stimulation of cultured hippocampal neurons produced AIS relocation of ankyrin $\mathrm{G}$ immunoreactivity only when the flashes were grouped into bursts (five flashes at $20 \mathrm{~Hz}$ every $5 \mathrm{~s}$ ), keeping the overall $1 \mathrm{~Hz}$ stimulation frequency constant (Grubb and Burrone, 2010). Interestingly, the effects of $1 \mathrm{~Hz}$ burst photostimulation were partially blocked by TTX (Grubb and Burrone, 2010). It was therefore suggested that high-frequency bursts of activity in hippocampal excitatory neurons can produce AIS relocation while steady stimulation at the same overall frequency does not (Grubb and Burrone, 2010).

The chronic M-channel inhibition also triggered a slow homeostatic adaptation of the E/I balance by inducing alterations 


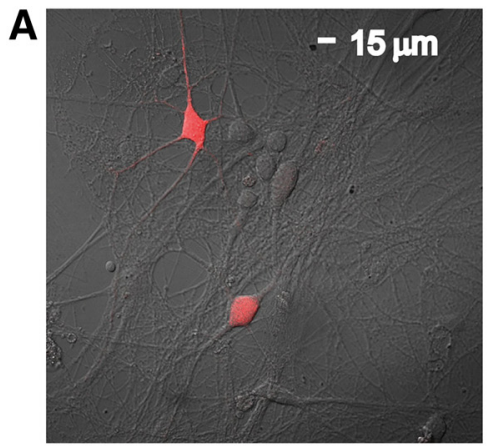

D

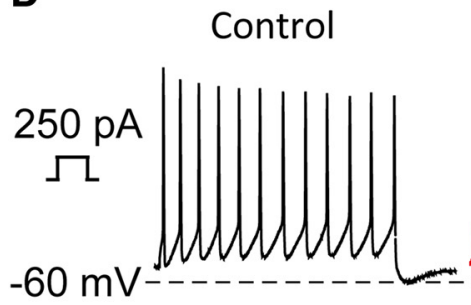

acute XE991
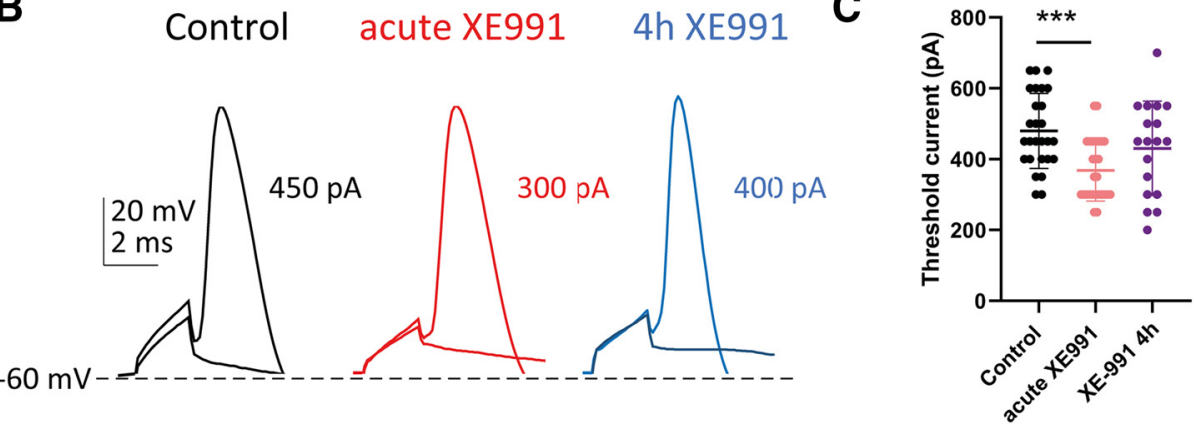

F

Control

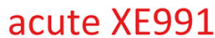

4h XE991

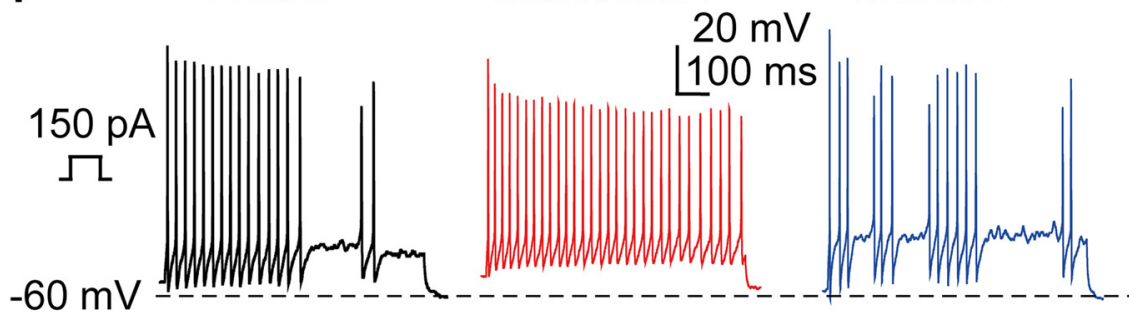

H
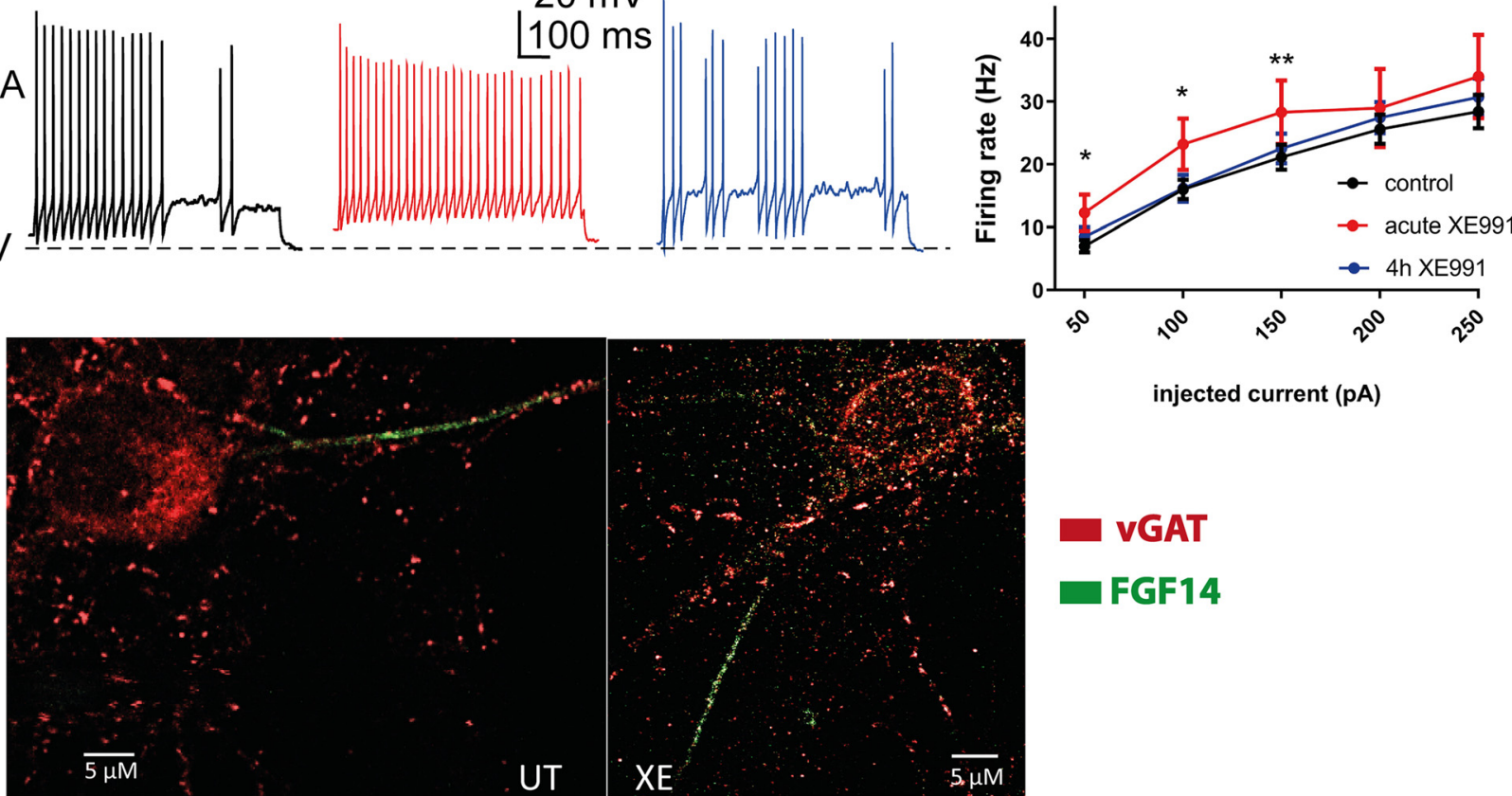

injected current (pA)
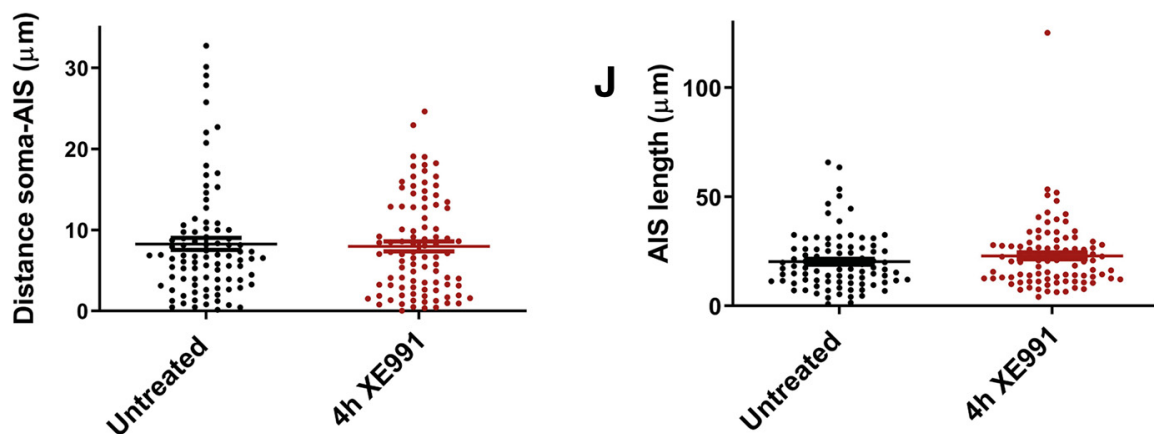

Figure 6. Lack of homeostatic plasticity at the level of intrinsic excitability in GABAergic inhibitory neurons following chronic XE991 treatment. $\boldsymbol{A}$, Red fluorescence identification of GABAergic inhibitory neurons in primary hippocampal cultures (14 DIV) by infection with the recombinant AAV derived from the AAV vector driving the expression of the fluorescent protein mCherry under the control of the specific GABAergic mDlx enhancer. $\boldsymbol{B}$, Representative traces of solitary spike discharge evoked by 2 ms step injection of depolarizing currents with increments of $25 \mathrm{pA}$ in the presence of synaptic blockers: in control conditions (black trace) and on acute XE991 exposure (red trace), as well as following $4 \mathrm{~h}$ exposure to XE991 (blue trace). $\boldsymbol{C}$, Acute 


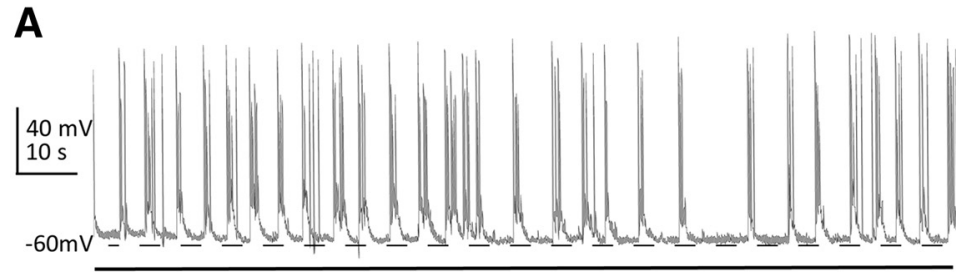

Control

B

B Untreated

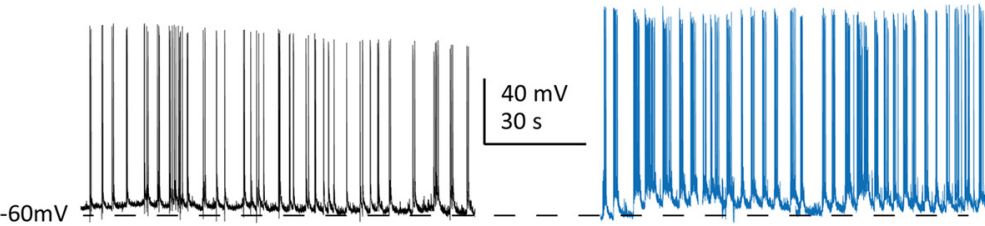

$48 \mathrm{~h}$ XE991

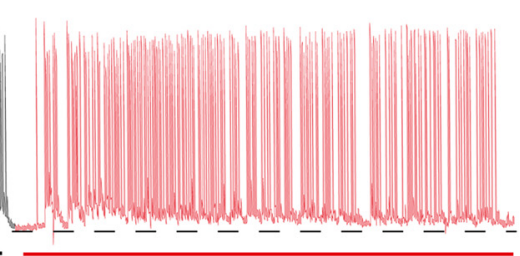

Acute XE991

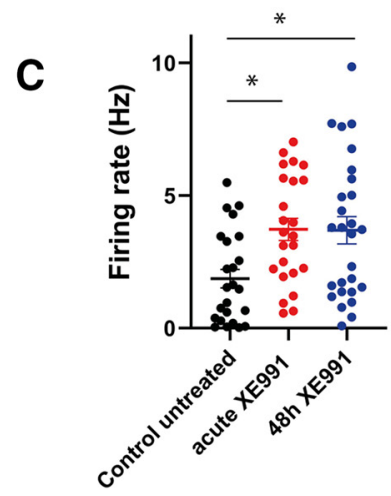

Figure 7. Lack of homeostatic plasticity at the level of spontaneous MFR in GABAergic inhibitory neurons following chronic XE991 treatment. $\boldsymbol{A}$, Representative traces showing the larger spontaneous firing rate after acute XE991 treatment (red trace) compared with control (black trace). B, Representative traces showing the higher spontaneous firing rate after $48 \mathrm{~h}$ XE991 treatment (blue trace) compared with untreated neurons (black trace). C, A significant increase in the ongoing MFR rate was found following acute M-channel inhibition, which was still observed

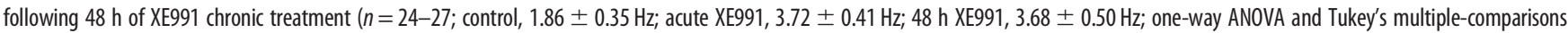
test: $p=0.0048, F_{(2,72)}=5.754$; control vs acute, $p=0.0122$; control vs $\left.48 \mathrm{~h} \mathrm{XE991,} p=0.0122\right)$. ${ }^{*} p<0.013$.

XE991 exposure significantly decreased the threshold current in GABAergic neurons ( $n=27$; control, $480 \pm 20$ pA; acute XE991, $369 \pm 17$ pA; one-way ANOVA, Tukey's multiple-comparisons test: $\left.p=0.0013, F_{(2,69)}=7.324\right)$. Following $4 \mathrm{~h}$ incubation with XE991, the threshold current was not significantly different from that of the acute treatment $(n=18 ; 4 \mathrm{~h}$ XE991, $431 \pm 31 \mathrm{pA}$; one-way ANOVA, $p=0.1441) .{ }^{* * *} p=0.0008$. $D$, Representative spike discharge in GABAergic neurons evoked by $400 \mathrm{~ms}$ step injection of $250 \mathrm{pA}$ depolarizing current in the presence of synaptic blockers in control conditions (black trace) and on acute XE991 exposure (red trace), as well as following $4 \mathrm{~h}$ exposure to XE991 (blue trace). $\boldsymbol{E}, \mathrm{F}-\mathrm{I}$ curves in GABAergic neurons. Acute XE991 (10 $\mu \mathrm{m})$ exposure significantly increased the frequency of evoked spike discharge ( $n=26$; Bonferroni multiple-comparison test, control vs acute: $250 \mathrm{pA}:{ }^{* *} p=0.0036$, $t=3.209, \mathrm{df}=25 ; 300 \mathrm{pA}:{ }^{* *} p=0.0042, t=3.151, \mathrm{df}=25 ; 350 \mathrm{pA}:{ }^{*} p=0.0168, t=2.562$, $\mathrm{df}=25 ; 400 \mathrm{pA}:{ }^{*} p=0.0191, t=2.549, \mathrm{df}=20$ ). However, following $4 \mathrm{~h}$ incubation with XE991, the F-I curve still showed increased frequency of spike discharge and was not significantly different from acute XE991 treatment $(n=20-26$, two-tailed unpaired $t$ test, where multiple comparisons were adjusted with multiple $t$ test Bonferroni correction; acute vs $4 \mathrm{~h}$ : $150 \mathrm{pA}: p=0.6136, t=0.5088, \mathrm{df}=41 ; 200 \mathrm{pA}: p=0.6117, t=0.5112 \mathrm{df}=44 ; 250 \mathrm{pA}$ : $p=0.6625, \quad t=0.4394, \quad \mathrm{df}=44 ; 300 \mathrm{pA}: \quad p=0.9546, \quad t=0.05,730, \quad \mathrm{df}=44 ; 350 \mathrm{pA}$ : $p=0.9136, t=0.1092, \mathrm{df}=42 ; 400 \mathrm{pA}: p=0.3126, t=1.025, \mathrm{df}=34 ; 450 \mathrm{pA}: p=0.5447$, $t=0.6174 \mathrm{df}=18) . \boldsymbol{F}$, Representative spike discharge in pyramidal-like excitatory neurons evoked by 400 ms step injection of $150 \mathrm{pA}$ depolarizing current in the presence of synaptic blockers in control conditions (black trace) and on acute XE991 exposure (red trace), as well as following $4 \mathrm{~h}$ exposure to XE991 (blue trace). G, F-I curves in pyramidal-like excitatory neurons. Acute XE991 exposure significantly increased the frequency of evoked spike discharge but sustained block of M-channels ( $4 \mathrm{~h}$ XE991) led to rapid adaptive changes that brought the values back to those of untreated neurons ( $n=47-68$; Bonferroni multiple-comparison test, control vs acute: $50 \mathrm{pA}:{ }^{*} p=0.0137, t=2.985, \mathrm{df}=10 ; 100 \mathrm{pA}:{ }^{*} p=0.0166, t=2.863, \mathrm{df}=10 ; 150 \mathrm{pA}$ : ${ }^{* *} p=0.0074, t=3.348, \mathrm{df}=10$; control vs $4 \mathrm{~h}$ XE991: $50 \mathrm{pA}: p=0.3988, t=0.8470$, $\mathrm{df}=113 ; \quad 100 \mathrm{pA}: \quad p=0.9400, \quad t=0.07,548, \mathrm{df}=113 ; 150 \mathrm{pA}: \quad p=0.6537, \quad t=0.4498$, $\mathrm{df}=112 ; 200 \mathrm{pA}: p=0.5964, t=0.5311, \mathrm{df}=113 ; 250 \mathrm{pA}: p=0.5722, t=0.5665, \mathrm{df}=113$ ). $\boldsymbol{H}$, Representative AIS FGF14 (green) and vGAT (red) coimmunostaining labels in GABAergic neurons. I, Following $4 \mathrm{~h}$ XE991 treatment, no changes were observed in the distance between the soma and the proximal boundary of the FGF14-immunoreactive signal ( $n=90-93$; unpaired two-tailed $t$ test: $p=0.7632, t=0.3017$, $\mathrm{df}=181$ ). J, Following $4 \mathrm{~h}$ XE991 treatment, no changes were observed in the total length of the FGF14-immunoreactive segment $(n=90$ 93; unpaired two-tailed $t$ test: $p=0.2049, t=1.272, \mathrm{df}=181$ ). in excitatory transmission. The homeostatic normalization of the E/I balance to chronic M-channel blockade occurred at a timescale of days, mainly due to compensations in sEPSCs rather than alterations in sIPSCs. The initial increase in synaptic excitatory output and its subsequent slow homeostatic adaptation is in line with the rise and the subsequent slow decrease of the MFR. The decrease in mEPSCs amplitude after $48 \mathrm{~h}$ of XE991 exposure suggests that the homeostasis involves slow adaptive changes in excitatory transmission. The paradoxical mismatch of the different plasticity timescales suggests that the fast $(\sim 4 \mathrm{~h})$ intrinsic homeostatic plasticity is not sufficient to account for the slow $(\sim 2 \mathrm{~d})$ homeostatic normalization of the network MFR. Our results show that in sharp contrast to pyramidal excitatory neurons, homeostatic plasticity of intrinsic excitability and of spontaneous MFR failed in GABAergic neurons of the same hippocampal cultures. Hence, inhibitory neurons remained hyperexcitable following chronic exposure to the M-channel blocker XE991 (Figs. 6, 7). Our data are in line with an in vivo study in the primary visual cortex showing homeostatic compensation at the level of spontaneous excitatory activity, but not in inhibitory neurons following enucleation (Barnes et al., 2015). Previous work suggests that the loss of M-channel function in GABAergic interneurons elevates their excitability (Lawrence et al., 2006), in line with our current results. Importantly, a recent study showed that selective deletion of M-channels $\left(\mathrm{K}_{\mathrm{v}} 7.2 / \mathrm{K}_{\mathrm{v}} 7.3\right)$ in parvalbumin GABAergic neurons leads to a compensatory increase of the fast excitatory transmission in pyramidal neurons (Soh et al., 2018). In addition, parvalbumin $\mathrm{K}_{\mathrm{v}} 7.2$-null mice showed increased seizure susceptibility, suggesting that decreases in M-channel activity in inhibitory neurons remodels the excitatory networks (Soh et al., 2018). Importantly, a substantial subpopulation of inhibitory neurons [e.g., VIP (vasoactive intestinal peptide-expressing) interneurons] has long been recognized as a potential disinhibitory circuit motif in the hippocampus. Since 
the Dlx promoter used to detect inhibitory neurons in our hippocampal culture restricts reporter expression to all types of GABAergic neurons (Dimidschstein et al., 2016), future studies are needed to identify the specific interneuron type lacking homeostatic adaptation.

Together, the data of the present study show that M-channel blockade triggers, at different temporal scales, the synaptic and intrinsic homeostatic plasticity of hippocampal excitatory neurons and that the latter process requires ongoing spiking activity. Although the nature of the interactions between the two plasticity processes is unknown, we speculate that the transient increase in spiking activity of hippocampal pyramidal neurons may trigger the fast adaptive changes in intrinsic excitability. Remarkably, homeostatic plasticity of intrinsic excitability and of MFR failed in hippocampal GABAergic inhibitory neurons in response to $\mathrm{M}$ channel inhibition, indicating that interneurons are vulnerable to this specific activity perturbation. In this context, the persistent hyperexcitability of GABAergic neurons may have a significant impact on network MFR. In all, our results indicate that the fast adaptation of intrinsic excitability in excitatory neurons is not sufficient to normalize the MFR, and that a single perturbation like M-channel inhibition can trigger multiple homeostatic mechanisms, which operate at different timescales to maintain the network mean firing rate.

\section{References}

Abbott LF, Nelson SB (2000) Synaptic plasticity: taming the beast. Nat Neurosci 3 [Suppl]:1178-1183.

Azouz R, Jensen MS, Yaari Y (1996) Ionic basis of spike after-depolarization and burst generation in adult rat hippocampal CA1 pyramidal cells. J Physiol 492:211-223.

Barnes SJ, Sammons RP, Jacobsen RI, Mackie J, Keller GB, Keck T (2015) Subnetwork-specific homeostatic plasticity in mouse visual cortex in vivo. Neuron 86:1290-1303.

Brown DA, Adams PR (1980) Muscarinic suppression of a novel voltage-sensitive $\mathrm{K}+$ current in a vertebrate neurone. Nature 283:673-676.

Bulow P, Murphy TJ, Bassell GJ, Wenner P (2019) Homeostatic intrinsic plasticity is functionally altered in Fmr1 KO cortical neurons. Cell Rep 26:1378-1388.e3.

Burrone J, O'Byrne M, Murthy VN (2002) Multiple forms of synaptic plasticity triggered by selective suppression of activity in individual neurons. Nature 420:414-418.

Chen S, Yaari Y (2008) Spike Ca2+ influx upmodulates the spike afterdepolarization and bursting via intracellular inhibition of KV7/M channels. J Physiol 586:1351-1363.

Davis GW (2006) Homeostatic control of neural activity: from phenomenology to molecular design. Annu Rev Neurosci 29:307-323.

Delmas P, Brown DA (2005) Pathways modulating neural KCNQ/M (Kv7) potassium channels. Nat Rev Neurosci 6:850-862.

Desai NS (2003) Homeostatic plasticity in the CNS: synaptic and intrinsic forms. J Physiol Paris 97:391-402.

Desai NS, Rutherford LC, Turrigiano GG (1999) Plasticity in the intrinsic excitability of cortical pyramidal neurons. Nat Neurosci 2:515-520.

Desai NS, Cudmore RH, Nelson SB, Turrigiano GG (2002) Critical periods for experience-dependent synaptic scaling in visual cortex. Nat Neurosci 5:783-789.

Dimidschstein J, Chen Q, Tremblay R, Rogers SL, Saldi G-A, Guo L, Xu Q, Liu R, Lu C, Chu J, Grimley JS, Krostag A-R, Kaykas A, Avery MC, Rashid MS, Baek M, Jacob AL, Smith GB, Wilson DE, Kosche G, et al. (2016) A viral strategy for targeting and manipulating interneurons across vertebrate species. Nat Neurosci 19:1743-1749.

French CR, Sah P, Buckett KJ, Gage PW (1990) A voltage-dependent persistent sodium current in mammalian hippocampal neurons. J Gen Physiol 95:1139-1157.

Goel A, Lee HK (2007) Persistence of experience-induced homeostatic synaptic plasticity through adulthood in superficial layers of mouse visual cortex. J Neurosci 27:6692-6700.
Golomb D, Yue C, Yaari Y (2006) Contribution of persistent Na+ current and M-type $\mathrm{K}+$ current to somatic bursting in CA1 pyramidal cells: combined experimental and modeling study. J Neurophysiol 96:19121926.

Grubb MS, Burrone J (2010) Activity-dependent relocation of the axon initial segment fine-tunes neuronal excitability. Nature 465:10701074.

Halliwell JV, Adams PR (1982) Voltage-clamp analysis of muscarinic excitation in hippocampal neurons. Brain Res 250:71-92.

Jentsch TJ (2000) Neuronal KCNQ potassium channels: physiology and role in disease. Nat Rev Neurosci 1:21-30.

Joseph A, Turrigiano GG (2017) All for one but not one for all: excitatory synaptic scaling and intrinsic excitability are coregulated by CaMKIV, whereas inhibitory synaptic scaling is under independent control. J Neurosci 37:6778-6785.

Karmarkar UR, Buonomano DV (2006) Different forms of homeostatic plasticity are engaged with distinct temporal profiles. Eur J Neurosci 23:1575-1584.

Kole MH, Brette R (2018) The electrical significance of axon location diversity. Curr Opin Neurobiol 51:52-59.

Lawrence JJ, Saraga F, Churchill JF, Statland JM, Travis KE, Skinner FK, McBain CJ (2006) Somatodendritic Kv7/KCNQ/M channels control interspike interval in hippocampal interneurons. J Neurosci 26:1232512338.

Lezmy J, Lipinsky M, Khrapunsky Y, Patrich E, Shalom L, Peretz A, Fleidervish IA, Attali B (2017) M-current inhibition rapidly induces a unique CK2-dependent plasticity of the axon initial segment. Proc Natl Acad Sci U S A 114:E10234-E10243.

Maffei A, Turrigiano G (2008) The age of plasticity: developmental regulation of synaptic plasticity in neocortical microcircuits. Prog Brain Res 169:211-223.

Maffei A, Nelson SB, Turrigiano GG (2004) Selective reconfiguration of layer 4 visual cortical circuitry by visual deprivation. Nat Neurosci 7:13531359.

Marder E, Goaillard JM (2006) Variability, compensation and homeostasis in neuron and network function. Nat Rev Neurosci 7:563-574.

Milshtein-Parush H, Frere S, Regev L, Lahav C, Benbenishty A, BenEliyahu S, Goshen I, Slutsky I (2017) Sensory deprivation triggers synaptic and intrinsic plasticity in the hippocampus. Cereb Cortex 27:3457-3470.

Pablo JL, Pitt GS (2017) FGF14 is a regulator of KCNQ2/3 channels. Proc Natl Acad Sci U S A 114:154-159.

Shah MM, Mistry M, Marsh SJ, Brown DA, Delmas P (2002) Molecular correlates of the M-current in cultured rat hippocampal neurons. J Physiol 544:29-37.

Slomowitz E, Styr B, Vertkin I, Milshtein-Parush H, Nelken I, Slutsky M, Slutsky I (2015) Interplay between population firing stability and single neuron dynamics in hippocampal networks. Elife 4:e04378.

Soh H, Park S, Ryan K, Springer K, Maheshwari A, Tzingounis AV (2018) Deletion of KCNQ2/3 potassium channels from PV + interneurons leads to homeostatic potentiation of excitatory transmission. Elife 7: e38617.

Styr B, Slutsky I (2018) Imbalance between firing homeostasis and synaptic plasticity drives early-phase Alzheimer's disease. Nat Neurosci 21:463473.

Styr B, Gonen N, Zarhin D, Ruggiero A, Atsmon R, Gazit N, Braun G, Frere S, Vertkin I, Shapira I, Harel M, Heim LR, Katsenelson M, Rechnitz O, Fadila S, Derdikman D, Rubinstein M, Geiger T, Ruppin E, Slutsky I (2019) Mitochondrial regulation of the hippocampal firing rate set point and seizure susceptibility. Neuron 102:1009-1024.e8.

Su H, Alroy G, Kirson ED, Yaari Y (2001) Extracellular calcium modulates persistent sodium current-dependent burst-firing in hippocampal pyramidal neurons. J Neurosci 21:4173-4182.

Turrigiano G (2011) Too many cooks? Intrinsic and synaptic homeostatic mechanisms in cortical circuit refinement. Annu Rev Neurosci 34:89103.

Turrigiano G (2012) Homeostatic synaptic plasticity: local and global mechanisms for stabilizing neuronal function. Cold Spring Harb Perspect Biol 4:a005736.

Turrigiano GG (2017) The dialectic of Hebb and homeostasis. Philos Trans R Soc Lond B Biol Sci 372:20160258. 
Turrigiano GG, Nelson SB (2004) Homeostatic plasticity in the developing nervous system. Nat Rev Neurosci 5:97-107.

Turrigiano GG, Leslie KR, Desai NS, Rutherford LC, Nelson SB (1998) Activity-dependent scaling of quantal amplitude in neocortical neurons. Nature 391:892-896.

Vertkin I, Styr B, Slomowitz E, Ofir N, Shapira I, Berner D, Fedorova T, Laviv T, Barak-Broner N, Greitzer-Antes D, Gassmann M, Bettler B, Lotan I, Slutsky I (2015) GABAB receptor deficiency causes failure of neuronal homeostasis in hippocampal networks. Proc Natl Acad Sci U S A 112:E3291-E3299.

Wefelmeyer W, Puhl CJ, Burrone J (2016) Homeostatic plasticity of subcellular neuronal structures: from inputs to outputs. Trends Neurosci 39:656-667.
Yin J, Yuan Q (2014) Structural homeostasis in the nervous system: a balancing act for wiring plasticity and stability. Front Cell Neurosci 8:439.

Yoshimura T, Rasband MN (2014) Axon initial segments: diverse and dynamic neuronal compartments. Curr Opin Neurobiol 27:96-102.

Yue C, Yaari Y (2004) KCNQ/M channels control spike afterdepolarization and burst generation in hippocampal neurons. J Neurosci 24:4614-4624.

Yue C, Yaari Y (2006) Axo-somatic and apical dendritic Kv7/M channels differentially regulate the intrinsic excitability of adult rat CA1 pyramidal cells. J Neurophysiol 95:3480-3495.

Yue C, Remy S, Su H, Beck H, Yaari Y (2005) Proximal persistent Na+ channels drive spike afterdepolarizations and associated bursting in adult CA1 pyramidal cells. J Neurosci 25:9704-9720. 